CONFORMAL GEOMETRY AND DYNAMICS

An Electronic Journal of the American Mathematical Society

Volume 10, Pages 63-99 (March 22, 2006)

S $1088-4173(06) 00126-3$

\title{
EXPANSION COMPLEXES FOR FINITE SUBDIVISION RULES. I
}

\author{
J. W. CANNON, W. J. FLOYD, AND W. R. PARRY
}

\begin{abstract}
This paper develops the basic theory of conformal structures on finite subdivision rules. The work depends heavily on the use of expansion complexes, which are defined and discussed in detail. It is proved that a finite subdivision rule with bounded valence and mesh approaching 0 is conformal (in the combinatorial sense) if there is a partial conformal structure on the model subdivision complex with respect to which the subdivision map is conformal. This gives a new approach to the difficult combinatorial problem of determining when a finite subdivision rule is conformal.
\end{abstract}

\section{INTRODUCTION}

A finite subdivision rule $\mathcal{R}$ essentially consists of a finite 2-dimensional $\mathrm{CW}$ complex $S_{\mathcal{R}}$ (called the model subdivision complex), a subdivision $\mathcal{R}\left(S_{\mathcal{R}}\right)$ of $S_{\mathcal{R}}$, and a continuous map $\sigma_{\mathcal{R}}: \mathcal{R}\left(S_{\mathcal{R}}\right) \rightarrow S_{\mathcal{R}}$ (called the subdivision map) which maps each open cell of $\mathcal{R}\left(S_{\mathcal{R}}\right)$ homeomorphically onto an open cell of $S_{\mathcal{R}}$. Since $S_{\mathcal{R}}$ is a CW complex, each 2-cell in $S_{\mathcal{R}}$ is the image of a 2-disk under an attaching map. These 2-disks are called the tile types of $\mathcal{R}$. If $\mathcal{R}$ is a finite subdivision rule, an $\mathcal{R}$-complex is essentially a 2-dimensional CW complex $X$ together with a continuous map $f: X \rightarrow S_{\mathcal{R}}$, called a structure map, which maps each open cell of $X$ homeomorphically onto an open cell of $S_{\mathcal{R}}$. In this case one can pull back the cell structure on $\mathcal{R}\left(S_{\mathcal{R}}\right)$ to get a subdivision $\mathcal{R}(X)$ of $X$ which is an $\mathcal{R}$-complex with structure map $\sigma_{\mathcal{R}} \circ f$. One can continue inductively to construct a sequence $\left\{\mathcal{R}^{n}(X)\right\}$ of subdivisions of $X$.

Our interest in finite subdivision rules comes from our ongoing attempt to resolve the following conjecture.

Conjecture 1.1. Suppose $G$ is a Gromov hyperbolic discrete group whose space at infinity $\partial G$ is the 2-sphere. Then $G$ acts properly discontinuously, cocompactly, and isometrically on hyperbolic 3-space $\mathbb{H}^{3}$.

Suppose $G$ is a Gromov hyperbolic group such that $\partial G$ is the 2-sphere. From a Cayley graph for $G$, one can define a sequence $\{\mathcal{D}(n)\}$ of shinglings (coverings

Received by the editors November 22, 2004.

2000 Mathematics Subject Classification. Primary 30F45, 52C20; Secondary 20F67, 52C26.

Key words and phrases. Conformality, expansion complex, finite subdivision rule.

This research was supported in part by NSF grants DMS-9803868, DMS-9971783, DMS10104030, and DMS-0203902.

(C)2006 American Mathematical Society Reverts to public domain 28 years from publication 
by compact, connected sets) of $\partial G$. Each $\mathcal{D}(n)$ is a finite cover of disks at infinity, each of which corresponds essentially to points closer to the tail of a geodesic ray than to the head of the ray; see $[8]$ for precise definitions. In [8, Theorem 2.3.1], Cannon and Swenson prove that $G$ acts properly discontinuously, cocompactly, and isometrically on hyperbolic 3 -space if and only if the sequence $\{\mathcal{D}(n)\}$ of disk covers is conformal in the sense of Cannon's combinatorial Riemann mapping theorem [3]. The definition of conformal from [3] has two axioms, but in 4 for the setting above those two axioms are replaced by a single axiom which is easily implied by either of them. Cannon and Swenson also show in [8] that for every integer $n \geq 2$ the elements of $\mathcal{D}(n)$ can be obtained from the elements of $\mathcal{D}(n-1)$ by a finite recursion.

Finite subdivision rules were developed to give models for the above sequences of disk covers. While finite subdivision rules are not as general as the subdivision rules that will probably be needed for this approach to Conjecture 1.1, they give a good starting point for first developing the theory. The basic theory of finite subdivision rules is given in [5, and techniques are developed there for determining when the sequence of subdivisions of a planar $\mathcal{R}$-complex is conformal. The reduction of the axioms of conformality to a single axiom that was given in [4] (in the context of the shinglings from half-spaces at infinity for the group graph of a Gromov hyperbolic group) was first proved for a large class of finite subdivision rules, and then the proof was modified for shinglings coming from Gromov hyperbolic groups.

Conjecture 1.1 is closely related to Thurston's Hyperbolization Conjecture, which states that if $M$ is a closed 3-manifold whose fundamental group is infinite, is not a free product, and does not contain a subgroup isomorphic to $\mathbb{Z} \oplus \mathbb{Z}$, then $M$ admits a hyperbolic structure. The Hyperbolization Conjecture is part of Thurston's Geometrization Conjecture, which states that every closed 3-manifold can be canonically decomposed into geometric pieces. In a series [17, 18, 19] of preprints, Perelman has announced a positive solution to Thurston's Geometrization Conjecture.

Note that by a theorem of Gromov [14, the hypotheses of Conjecture 1.1 are satisfied by the fundamental group of a closed 3-manifold of negative Riemannian sectional curvature. If Conjecture 1.1 were true for such a 3-manifold, then it would follow from a theorem of Gabai, Meyerhoff, and N. Thurston [12] that the manifold has a hyperbolic structure. It follows from a theorem of Bestvina and Mess [1 that if the fundamental group $G$ of a closed 3-manifold is Gromov hyperbolic, then $\partial G$ is a 2-sphere. Hence the Hyperbolization Conjecture would follow from Conjecture 1.1 and Mosher's Weak Hyperbolization Conjecture [15], which states that if $M$ is a closed 3-manifold whose fundamental group is infinite, is not a free product, and does not contain a subgroup isomorphic to $\mathbb{Z} \oplus \mathbb{Z}$, then the fundamental group is Gromov hyperbolic.

We develop here a new method for proving conformality of finite subdivision rules. This method seems very promising, and is central to our approach to Conjecture 1.1. We define partial conformal structures on subdivision complexes of finite subdivision rules ("partial" because they are not defined on the vertices or on open edges in the boundary), and prove that a finite subdivision rule $\mathcal{R}$ with bounded valence and mesh approaching 0 is conformal if there is a conformal structure on the model subdivision complex $S_{\mathcal{R}}$ with respect to which the subdivision map is conformal. This use of partial conformal structures was inspired by the 


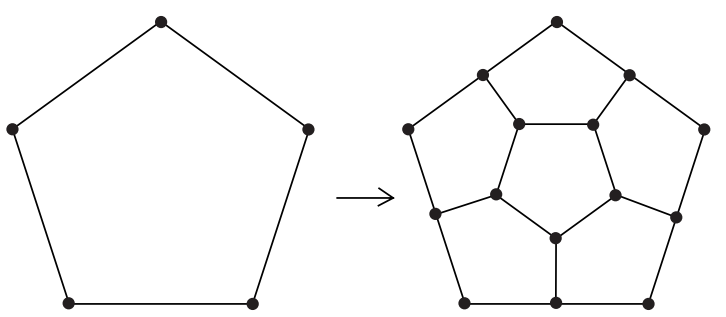

Figure 1. The subdivision of the tile type for the pentagonal subdivision rule

Bowers-Stephenson paper 2. Our work depends heavily on the use of expansion complexes. An expansion $\mathcal{R}$-complex (or, more simply, an expansion complex) for a finite subdivision rule $\mathcal{R}$ is an $\mathcal{R}$-complex $X$, with structure map $f: X \rightarrow S_{\mathcal{R}}$, which satisfies the following:

(i) $X$ is homeomorphic to $\mathbb{R}^{2}$; and

(ii) there is an orientation-preserving homeomorphism $\varphi: X \rightarrow X$ such that $\sigma_{\mathcal{R}} \circ f=f \circ \varphi$.

If $X$ is an expansion complex as above, then $\varphi$ is an $\mathcal{R}$-isomorphism from $\mathcal{R}(X)$ to $X$.

Expansion complexes arise naturally from direct limits. For example, Figure 1 shows the subdivision of the tile type for the pentagonal subdivision rule. The pentagon at the left of the figure can be identified with the central pentagon in the subdivision at the right of the figure. As described in Lemma 2.4, this generates a direct limit expansion complex. This expansion complex, which is shown in Figure 2. was studied by Bowers and Stephenson in [2]. They constructed a conformal structure on the expansion complex for which the expansion map is conformal and every tile is conformally regular.

Our use of expansion complexes and conformal structures for studying conformality of finite subdivision rules is influenced by Thurston's topological characterization theorem for critically finite branched maps (see, for example, 10] or [21]). If $f: S^{2} \rightarrow S^{2}$ is a critically finite branched map, then one can put an orbifold structure $\mathcal{O}_{f}$ on $S^{2}$ by assigning to a point $x \in S^{2}$ the least common multiple of the degrees of all preimages of $x$ under iterates of $f$. Furthermore, $f$ induces a map $\tau_{f}: \mathcal{T}\left(\mathcal{O}_{f}\right) \rightarrow \mathcal{T}\left(\mathcal{O}_{f}\right)$, where $\mathcal{T}\left(\mathcal{O}_{f}\right)$ is the Teichmüller space of $\mathcal{O}_{f}$. Thurston proved that $f$ can be realized by a rational map exactly if $\tau_{f}$ has a fixed point, and he then topologically characterized when $\tau_{f}$ has a fixed point. For each $f$-stable curve system $\Gamma$ on $\mathcal{O}_{f}$ one defines an associated matrix $A^{\Gamma}$ with nonnegative entries. In the typical case where the orbifold $\mathcal{O}_{f}$ is hyperbolic, $\tau_{f}$ has a fixed point exactly if the spectral radius of $A^{\Gamma}$ is less than 1 for every $f$-stable curve system $\Gamma$.

A connection between Thurston's characterization theorem and finite subdivision rules is given in [6] in joint work with Richard Kenyon. A finite subdivision rule has an edge pairing if the model subdivision complex $S_{\mathcal{R}}$ is a closed surface. If $S_{\mathcal{R}}$ is a connected orientable closed surface, then it follows from the Riemann-Hurwitz formula that either (i) $\sigma_{\mathcal{R}}$ is a homeomorphism (so tiles are not being properly subdivided), (ii) $S_{\mathcal{R}}$ is a torus and $\sigma_{\mathcal{R}}$ is a covering map, or (iii) $S_{\mathcal{R}}$ is a 2-sphere. In the latter case, $\sigma_{\mathcal{R}}$ is a critically finite branched map and one can use Thurston's characterization theorem to decide whether or not $\sigma_{\mathcal{R}}$ can be realized by a rational 


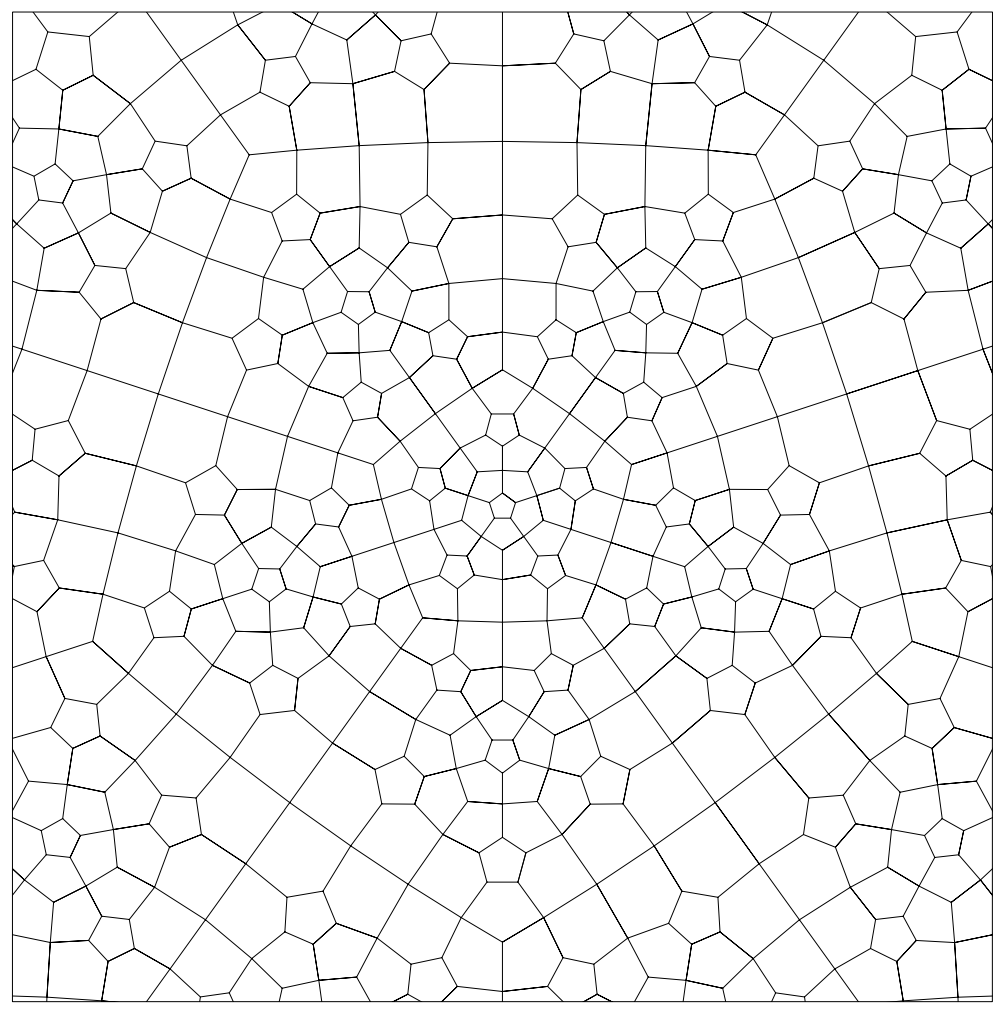

FiguRE 2. An expansion complex for the pentagonal subdivision rule

map. We prove in [6, Theorem 3.1] that if $\mathcal{R}$ is an orientation-preserving finite subdivision rule with an edge pairing such that $\mathcal{R}$ has bounded valence, $\mathcal{R}$ has mesh approaching $0, S_{\mathcal{R}}$ is a 2 -sphere, and $\mathcal{R}$ is conformal, then the subdivision map $\sigma_{\mathcal{R}}$ is realizable by a rational map.

Our hope is to reformulate Thurston's characterization theorem (at least for critically finite branched maps without periodic critical points) from the point of view of finite subdivision rules, and then to extend that theory to finite subdivision rules without edge pairings. We envision defining a Teichmüller space $\mathcal{T}\left(S_{\mathcal{R}}\right)$ for the model subdivision complex $S_{\mathcal{R}}$ of a finite subdivision rule $\mathcal{R}$ and then characterizing when there is a fixed point for the induced map $\tau_{\mathcal{R}}: \mathcal{T}\left(S_{\mathcal{R}}\right) \rightarrow \mathcal{T}\left(S_{\mathcal{R}}\right)$. In this paper we give technical underpinnings for the development of such a Teichmüller theory for finite subdivision rules. In particular we prove that a finite subdivision rule $\mathcal{R}$ with bounded valence and mesh approaching 0 is conformal if the model subdivision complex $S_{\mathcal{R}}$ has an invariant partial conformal structure.

In Section 2 we give basic definitions and examples for finite subdivision rules and expansion complexes, and we derive some basic properties of expansion complexes. This is followed in Section 3 by the definitions of partial conformal structures and conformal structures on model subdivision complexes. A partial conformal structure on a subdivision complex $X$ is given by an atlas of complex-valued charts, with conformal or anticonformal transition functions on open tiles and butterflies $(=$ open tile pairs). Such structures are called partial because they are not defined at 
the vertices of $X$ or at boundary points of $X$. A partial conformal structure on $S_{\mathcal{R}}$ is called invariant under subdivision, or simply invariant, if the subdivision map $\sigma_{\mathcal{R}}$ is conformal or anticonformal on the complement of the vertices and boundary points of $S_{\mathcal{R}}$ (conformal or anticonformal on open tiles and open tile pairs).

If $X$ is an $\mathcal{R}$-complex, then a partial conformal structure on $S_{\mathcal{R}}$ pulls back to a partial conformal structure on $\operatorname{int}(X)$. A partial conformal structure on $S_{\mathcal{R}}$ is called nonsingular if the induced partial conformal structure on $\operatorname{int}(X)$ extends across the vertices of $\operatorname{int}(X)$ to a conformal structure on all of $\operatorname{int}(X)$ whenever $X$ is a planar $\mathcal{R}$-complex.

The heart of the paper is Section 4 Theorem 4.2 shows that if a finite subdivision rule $\mathcal{R}$ has bounded valence and mesh approaching 0 , then every invariant partial conformal structure on $S_{\mathcal{R}}$ is nonsingular. We then give several theorems on the effects of partial conformal structures on the sizes and shapes of tiles. A key to this is a strong combinatorial finiteness statement. The bounded valence condition gives a local combinatorial finiteness for a subdivision complex, but this isn't enough to control shapes of tiles because closed tiles need not be contractible. Lemma $4.3 \mathrm{im}-$ plies that every expansion $\mathcal{R}$-complex $X$ for a finite subdivision rule with bounded valence and mesh approaching 0 has a subdivision with finitely many disk neighborhood types (we leave the definition of this to Section 4). Furthermore, if $X$ has an induced conformal structure from a partial conformal structure on $S_{\mathcal{R}}$, there are only finitely many disk neighborhood types up to conformal isomorphisms. Theorems 4.4. 4.5. and 4.6 use this to establish metric properties of tiles in expansion complexes. The proofs unexpectedly apply classical results on conformal mappings, including Koebe's distortion theorem and the $\frac{1}{4}$-theorem. The section culminates with Theorem 4.7, which states that a finite subdivision rule $\mathcal{R}$ is conformal if it has bounded valence, mesh approaching 0 , and an invariant partial conformal structure.

Suppose $X$ is an $\mathcal{R}$-complex and $X$ is homeomorphic to $\mathbb{R}^{2}$. We call a conformal structure on $X$ parabolic if its uniformization is $\mathbb{C}$ and hyperbolic if its uniformization is the disk. In Section 5 we give combinatorial descriptions (in terms of combinatorial moduli) for determining whether a conformal structure on $X$ is parabolic or hyperbolic. It follows from the descriptions that, if $\mathcal{R}$ has mesh approaching 0 , then $X$ is parabolic (respectively hyperbolic) if and only if $\mathcal{R}(X)$ is parabolic (respectively hyperbolic). (This is needed for the main application in [7].) Furthermore, whether a conformal structure on $X$ induced from a partial conformal structure on $S_{\mathcal{R}}$ is parabolic or hyperbolic is independent of the partial conformal structure on $S_{\mathcal{R}}$.

In Section 6 we give conditions under which an expansion map $\varphi$ is topologically conjugate to a linear map $\psi$ such that the combinatorial distance between $\varphi$ and $\psi$ is uniformly bounded. In particular, suppose $\mathcal{R}$ is a finite subdivision rule with bounded valence and mesh approaching 0 , and $X$ is an expansion $\mathcal{R}$-complex with underlying space $\mathbb{R}^{2}$ and with expansion map $\varphi$. Suppose further that $\psi: X \rightarrow$ $X$ is a conformal homeomorphism such that there is a global upper bound on $d(\varphi(x), \psi(x))$ for all $x \in X$, where $d(u, v)$ is the minimum length of a chain of tiles of $X$ which connects $u$ and $v$. Then it follows from Theorem 6.6 that $\mathbb{R}^{2}$ can be given an $\mathcal{R}$-complex structure $X^{\prime}$ such that $X^{\prime}$ is an expansion complex with expansion map $\psi$ and there is a topological conjugacy between $\varphi$ and $\psi$. The results in this section are important for applications and for the further development of the theory. 
In a companion paper [7] we give applications of the theory in this paper. The main application, and the one that motivated some of this work, is the theorem that a one-tile rotationally invariant finite subdivision rule is conformal. The proof uses much of the machinery of this paper. In a second application we consider a one-tile finite subdivision rule $\mathcal{R}$ with bounded valence and mesh approaching 0 . Let $t$ be a tile type of $\mathcal{R}$. We assume further that there is a constant $r$ such that, for every positive integer $n$ and every tile type $t$, every interior vertex of $\mathcal{R}^{n}(t)$ has valence $r$. We first prove that either $t$ is a triangle and $r=6, t$ is a quadrilateral and $r=4$, or $t$ is a hexagon and $r=3$. We then show that the expansion map of every $\mathcal{R}$-expansion complex can be conjugated to a linear map. We finally show using the results in Sections 4 and 6 that $\mathcal{R}$ is conformal exactly if this linear map is either a dilation or has eigenvalues that are not real.

Given an expansion complex, it can be surprisingly difficult to determine whether it is parabolic or hyperbolic. We give an interesting example of this difficulty in [7. We consider there an irreducible finite subdivision rule with bounded valence and mesh approaching 0 such that it has a hyperbolic expansion complex $X$ and a parabolic expansion complex $Y$. Furthermore, any compact subcomplex of $X$ (respectively $Y$ ) is isomorphic to a subcomplex of $Y$ (respectively $X$ ).

\section{Definitions, EXAMPLES AND First PROPERTiES}

We begin this section by recalling and expanding upon some of the basic definitions from Section 1.1 of [5. For us a CW complex is a topological space equipped with a distinguished cell structure, and cells of CW complexes are closed unless explicitly stated otherwise. On occasion we refer to the topological space as the underlying space of the CW complex.

A finite subdivision rule $\mathcal{R}$ consists of the following:

(1) A finite 2-dimensional CW complex $S_{\mathcal{R}}$, called the model subdivision complex, such that $S_{\mathcal{R}}$ is the union of its closed 2-cells. For each closed 2-cell $s$ of $S_{\mathcal{R}}$ there is a closed 2-disk $t$ and a characteristic map $f: t \rightarrow s$. We assume that $t$ has a CW complex structure such that $t$ has at least three vertices, the vertices and edges of $t$ are contained in $\partial t$, and the restriction of $f$ to every open cell of $t$ is a homeomorphism onto an open cell of $s$.

(2) A finite CW complex $\mathcal{R}\left(S_{\mathcal{R}}\right)$ which is a subdivision of $S_{\mathcal{R}}$. That is, $\mathcal{R}\left(S_{\mathcal{R}}\right)$ and $S_{\mathcal{R}}$ have the same underlying space and every cell of $\mathcal{R}\left(S_{\mathcal{R}}\right)$ is contained in a cell of $S_{\mathcal{R}}$.

(3) A continuous map $\sigma_{\mathcal{R}}: \mathcal{R}\left(S_{\mathcal{R}}\right) \rightarrow S_{\mathcal{R}}$, called the subdivision map, whose restriction to every open cell of $\mathcal{R}\left(S_{\mathcal{R}}\right)$ is a homeomorphism onto an open cell of $S_{\mathcal{R}}$.

If $s$ is a 2-cell in $S_{\mathcal{R}}$, then the CW complex $t$ in condition (1) is called a tile type. The tile type $t$ comes equipped with its characteristic map. Given an edge of $S_{\mathcal{R}}$, there exists a closed 1-disk $e$ equipped with a characteristic map $g: e \rightarrow S_{\mathcal{R}}$ which takes $e$ to the given edge. We call $e$ an edge type.

Suppose $\mathcal{R}$ is a finite subdivision rule. An $\mathcal{R}$-complex consists of a 2 -dimensional CW complex $X$ which is the union of its 2-cells and is equipped with a continuous map $f: X \rightarrow S_{\mathcal{R}}$ whose restriction to each open cell of $X$ is a homeomorphism 
onto an open cell of $S_{\mathcal{R}}$. We call $f$ the structure map of $X$. We call $X$ a subdivision complex when we do not wish to mention $\mathcal{R}$ explicitly. We refer to 0 -cells of subdivision complexes as vertices, to 1-cells as edges, and to 2-cells as tiles. A point $x$ of a subdivision complex $X$ is an interior point of $X$ if there is a continuous injection from $D=\{z \in \mathbb{C}:|z|<1\}$ to $X$ which maps 0 to $x$. The set of interior points of $X$ is called the interior of $X$ and is denoted $\operatorname{int}(X)$; its complement $X \backslash \operatorname{int}(X)$ is called the boundary of $X$. If a subdivision complex $X$ is not a surface, then its interior need not be an open subset of $X$.

Suppose that $\mathcal{R}$ is a finite subdivision rule and $X$ is an $\mathcal{R}$-complex with structure map $f: X \rightarrow S_{\mathcal{R}}$. The subdivision $\mathcal{R}\left(S_{\mathcal{R}}\right)$ of $S_{\mathcal{R}}$ pulls back via $f$ to a subdivision $\mathcal{R}(X)$ of $X$ so that $\mathcal{R}(X)$ is an $\mathcal{R}$-complex with structure map $\sigma_{\mathcal{R}} \circ f: \mathcal{R}(X) \rightarrow S_{\mathcal{R}}$. We can define $\mathcal{R}^{n}(X)$, for $n$ a nonnegative integer, recursively by $\mathcal{R}^{0}(X)=X$ and $\mathcal{R}^{n}(X)=\mathcal{R}\left(\mathcal{R}^{n-1}(X)\right)$ if $n \geq 1$.

Suppose that $\mathcal{R}$ is a finite subdivision rule, and $X$ and $X^{\prime}$ are $\mathcal{R}$-complexes with structure maps $f: X \rightarrow S_{\mathcal{R}}$ and $f^{\prime}: X^{\prime} \rightarrow S_{\mathcal{R}}$. A continuous cellular map $h: X \rightarrow X^{\prime}$ is called an $\mathcal{R}$-map if $f=f^{\prime} \circ h$. An $\mathcal{R}$-map that is a cellular isomorphism is an $\mathcal{R}$-isomorphism.

Now we proceed to some new definitions. Let $\mathcal{R}$ be a finite subdivision rule with model subdivision complex $S_{\mathcal{R}}$ and subdivision map $\sigma_{\mathcal{R}}: \mathcal{R}\left(S_{\mathcal{R}}\right) \rightarrow S_{\mathcal{R}}$. Let $X$ be an $\mathcal{R}$-complex with structure map $f: X \rightarrow S_{\mathcal{R}}$. We call $X$ an expansion $\mathcal{R}$-complex or simply an expansion complex if it satisfies the following:

(1) $X$ is homeomorphic to $\mathbb{R}^{2}$; and

(2) there is an orientation-preserving homeomorphism $\varphi: X \rightarrow X$ such that $\sigma_{\mathcal{R}} \circ f=f \circ \varphi$.

We call $\varphi$ an expansion map. Note that if $X$ is an expansion $\mathcal{R}$-complex and $Y=\mathcal{R}(X)$, then $\varphi$ is an $\mathcal{R}$-isomorphism from $Y$ to $X$. Furthermore, there is another $\mathcal{R}$-complex $W$ with the same underlying space such that $X=\mathcal{R}(W)$ and $\varphi$ is an $\mathcal{R}$-isomorphism from $X$ to $W$. The cells of $W$ are the images under $\varphi$ of the cells of $X$, and the structure map for $W$ is the map $f \circ \varphi^{-1}: W \rightarrow S_{\mathcal{R}}$.

We present some simple examples.

Example 2.1 (The pentagonal subdivision rule). This finite subdivision rule was considered in [2], 5], and [6. The model subdivision complex has one vertex, one edge, and one face. The tile type is a pentagon; it is subdivided into six pentagons as in Figure 1. Two expansion complexes for the pentagonal subdivision rule are shown in Figures 2 and 3 . Figures 2 and 3 were drawn using Ken Stephenson's program CirclePack [20, as was Figure 6. In each example $\varphi$ fixes the point $p$ at the center of the figure. In Figure $2 \operatorname{star}(p, X)$ is a single pentagon, and in Figure 3 $\operatorname{star}(p, X)$ is a union of four pentagons.

Example 2.2 (The binary quadrilateral subdivision rule). This finite subdivision rule was considered in [5] and [6]. The model subdivision complex is the torus $\mathbb{R}^{2} / \mathbb{Z}^{2}$, with the tiling induced from the square tiling of the plane. The subdivision map $\sigma_{\mathcal{R}}$ is the map on the torus induced from the map $(x, y) \mapsto(2 x, 2 y)$ on $\mathbb{R}^{2}$.

The single tile type of the binary quadrilateral subdivision rule is a quadrilateral; its subdivision is shown in Figure 4. An example of an expansion complex is the square tiling of the plane with structure map the quotient map $\mathbb{R}^{2} \rightarrow \mathbb{R}^{2} / \mathbb{Z}^{2}$ and with $\varphi$ the map $(x, y) \mapsto(2 x, 2 y)$. Note that $\varphi$ fixes the origin. 


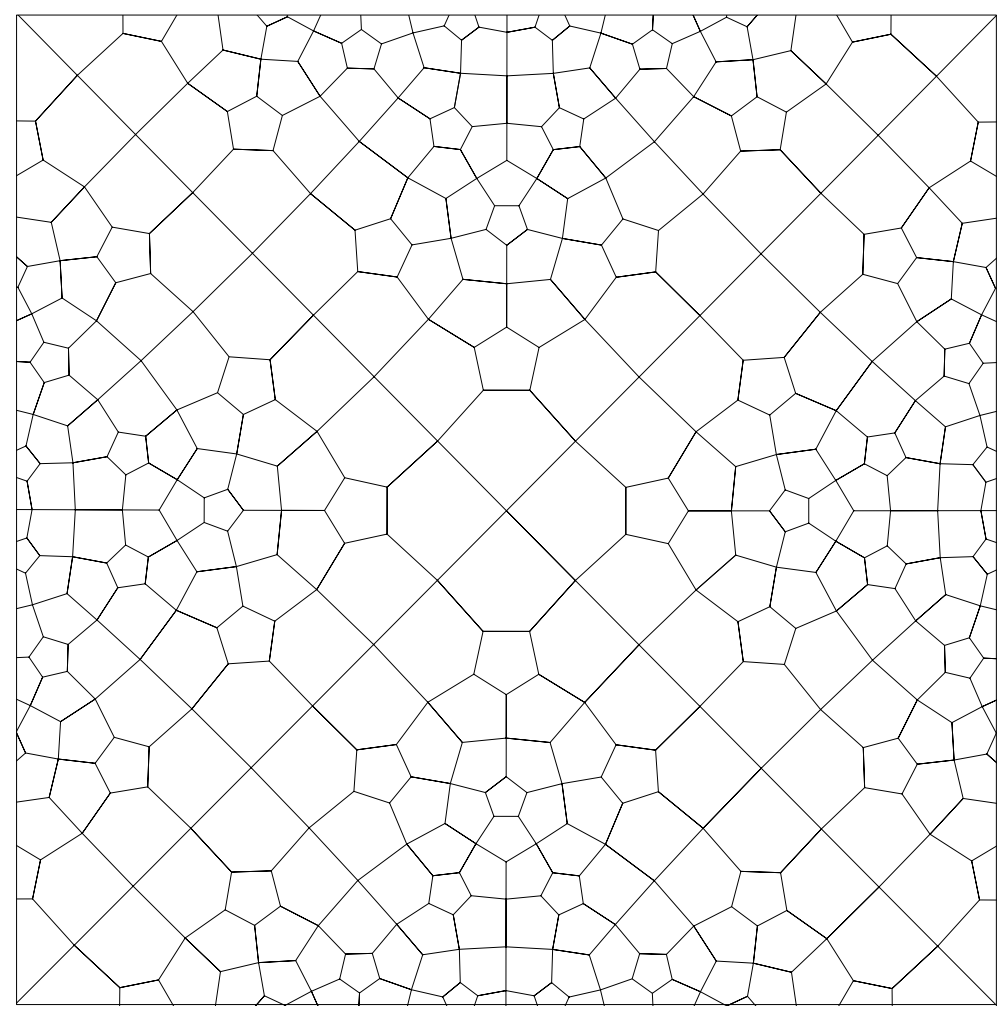

FiguRe 3. Another expansion complex for the pentagonal subdivision rule

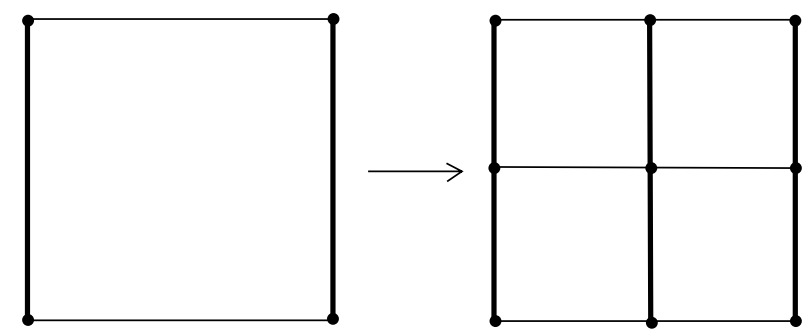

Figure 4. The subdivision of the tile type for the binary quadrilateral subdivision rule

Example 2.3 (The dodecahedral subdivision rule). This finite subdivision rule was considered in [5]. The model subdivision complex has one vertex, two edges, and three faces. The subdivisions of the three tile types are shown in Figure 5 . An expansion complex for the dodecahedral subdivision rule is shown in Figure 6, In this example the expansion map fixes a point in the interior of a pentagonal tile.

The following lemma gives a general construction for expansion complexes. We precede it with a definition. Let $\mathcal{R}$ be a finite subdivision rule, and let $X$ be an expansion $\mathcal{R}$-complex with expansion map $\varphi$. We say that an $\mathcal{R}$-subcomplex $S$ of $X$ is a seed of $X$ if $S$ is a closed topological disk, $S \subseteq \varphi(S)$, and $X=\bigcup_{n=0}^{\infty} \varphi^{n}(S)$. 


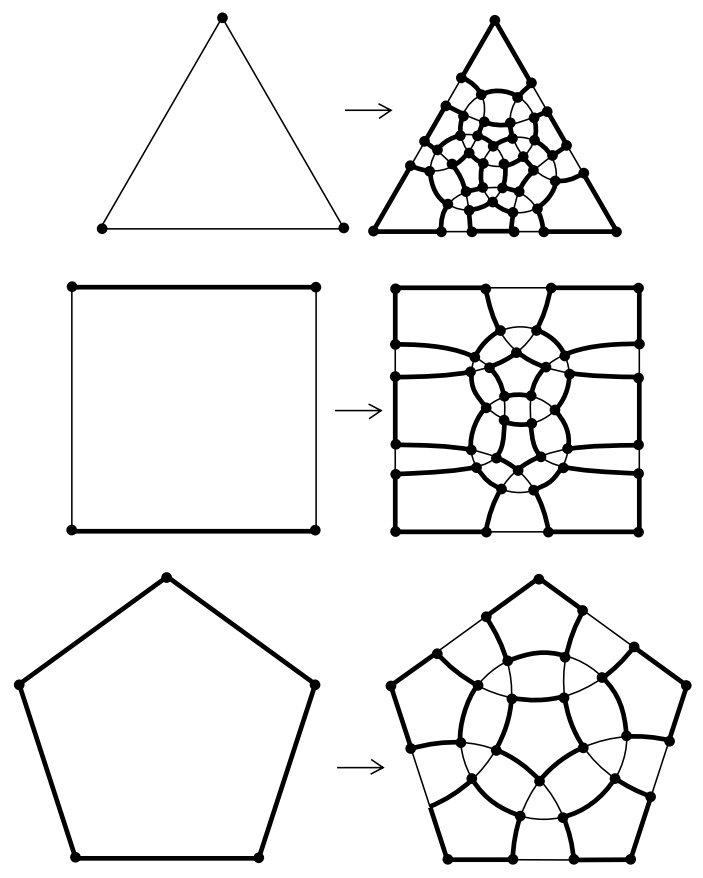

Figure 5. The subdivisions of the tile types for the dodecahedral subdivision rule

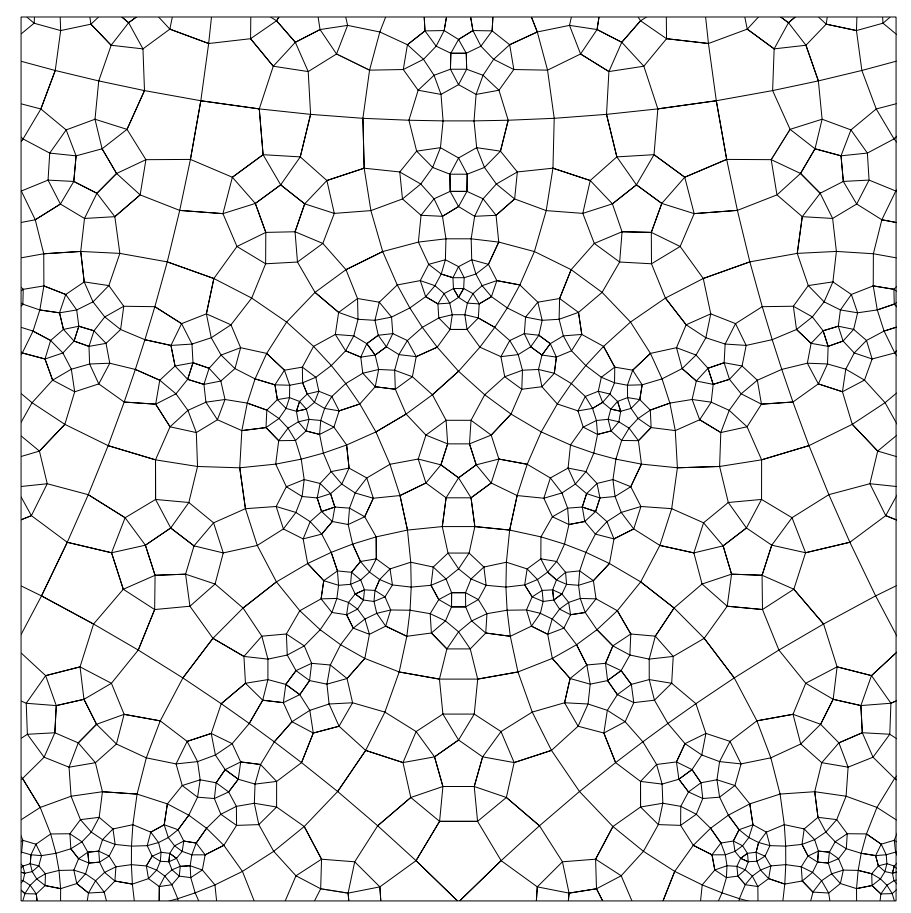

Figure 6. An expansion complex for the dodecahedral subdivision rule 

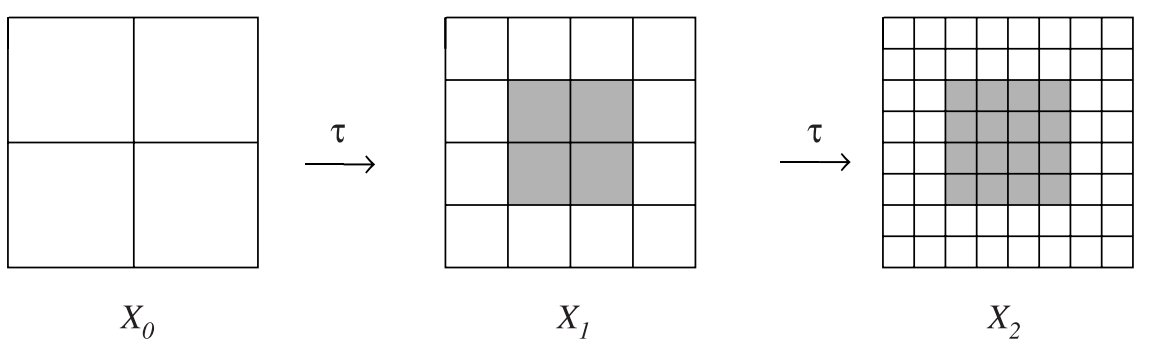

Figure 7. Constructing the direct limit $X$

Lemma 2.4. Let $\mathcal{R}$ be a finite subdivision rule. Suppose that $S$ is an $\mathcal{R}$-complex such that $S$ is a closed topological disk and there is an orientation-preserving $\mathcal{R}$ isomorphism from $S$ to a subcomplex of $\mathcal{R}(S)$ that is contained in the interior of $\mathcal{R}(S)$. Then there is an expansion $\mathcal{R}$-complex $X$ with a seed which is $\mathcal{R}$-isomorphic to $S$.

Proof. Let $X_{n}=\mathcal{R}^{n}(S)$ for every nonnegative integer $n$. By assumption there exists an orientation-preserving $\mathcal{R}$-map $\tau: X_{0} \rightarrow X_{1}$ which maps $X_{0}$ isomorphically onto a subcomplex of $X_{1}$ which is contained in the interior of $X_{1}$. For each nonnegative integer $n$ the map $\tau$ is an $\mathcal{R}$-map from $X_{n}$ to $X_{n+1}$. Figure 7 shows $\mathcal{R}$-complexes $X_{0}, X_{1}, X_{2}$, and the images $\tau\left(X_{0}\right)$ and $\tau\left(X_{1}\right)$ for the finite subdivision rule of Example 2.2, We obtain a directed system $X_{0} \stackrel{\tau}{\rightarrow} X_{1} \stackrel{\tau}{\rightarrow} X_{2} \stackrel{\tau}{\rightarrow} \cdots$. We denote its direct limit by $X$. It follows that $X$ is a 2-dimensional CW complex which is the union of its 2-cells and $X$ is homeomorphic to $\mathbb{R}^{2}$.

Let $f_{n}: X_{n} \rightarrow S_{\mathcal{R}}$ be the structure map of $X_{n}$ for every nonnegative integer $n$. Then $f_{n+1} \circ \tau=f_{n}$. Passing to the limit, we get a map $f: X \rightarrow S_{\mathcal{R}}$, which gives $X$ the structure of an $\mathcal{R}$-complex.

We next define an expansion map for $X$. Let id: $S \rightarrow S$ be the identity map. We have the following commutative diagram:

$$
\begin{array}{cccccccc} 
& & X_{0} & \stackrel{\tau}{\rightarrow} & X_{1} & \stackrel{\tau}{\rightarrow} & X_{2} & \stackrel{\tau}{\rightarrow} \\
& & \downarrow \text { id } & & \downarrow & & \\
X_{0} & \stackrel{\tau}{\rightarrow} & X_{1} & \stackrel{\tau}{\rightarrow} & X_{2} & \stackrel{\tau}{\rightarrow} & X_{3} & \stackrel{\tau}{\rightarrow}
\end{array}
$$

The vertical maps determine an orientation-preserving homeomorphism $\varphi: X \rightarrow$ $X$. Because $\sigma_{\mathcal{R}} \circ f_{n}=f_{n+1}$ for every nonnegative integer $n$, it follows that $\sigma_{\mathcal{R}} \circ f_{n}=$ $f_{n+1} \circ$ id for every nonnegative integer $n$. Passing to the limit, $\sigma_{\mathcal{R}} \circ f=f \circ \varphi$. Thus $X$ is an expansion $\mathcal{R}$-complex with expansion map $\varphi$.

Finally, one checks that $X$ contains a seed which is $\mathcal{R}$-isomorphic to $S$.

We next recall some more definitions from Section 1.1 of $[5$. Let $\mathcal{R}$ be a finite subdivision rule. We say that $\mathcal{R}$ has bounded valence if there is an upper bound on the vertex valences of the subdivisions $\mathcal{R}^{n}\left(S_{\mathcal{R}}\right)$ of the model subdivision complex $S_{\mathcal{R}}$ as $n$ varies over the nonnegative integers. We say that an $\mathcal{R}$-complex has bounded valence if there is an upper bound on the valences of its vertices. We say that the mesh of $\mathcal{R}$ approaches 0 if for every open cover of $S_{\mathcal{R}}$ there exists a positive integer $n$ such that every tile in $\mathcal{R}^{n}\left(S_{\mathcal{R}}\right)$ is contained in one of the sets of that open cover. Given a positive integer $N$, let $\mathcal{R}^{N}$ be the finite subdivision rule whose model subdivision complex equals the model subdivision complex of $\mathcal{R}$ and 
whose subdivision map is the $N$ th iterate of the subdivision map for $\mathcal{R}$. Without previous mention of $N$, by an expansion $\mathcal{R}^{N}$-complex we mean an expansion complex with respect to the finite subdivision rule $\mathcal{R}^{N}$ for some positive integer $N$.

Given a finite subdivision rule $\mathcal{R}$, one might ask whether there exists an $\mathcal{R}$ complex $S$ as in Lemma 2.4. The next result states that there are enough such $\mathcal{R}$-complexes for our purposes.

Lemma 2.5. Let $\mathcal{R}$ be a finite subdivision rule with bounded valence and mesh approaching 0 . Suppose that $X$ is a surface which is an $\mathcal{R}$-complex with a point $p$ in its interior. Then there exists an $\mathcal{R}$-subcomplex $S$ of some subdivision of $X$ such that $p \in \operatorname{int}(S)$ and $S$ is $\mathcal{R}$-isomorphic to a seed of an expansion $\mathcal{R}^{N}$-complex.

Proof. Let $Y_{1}=\operatorname{star}(p, X)$. Because the mesh of $\mathcal{R}$ approaches 0 , there exists a positive integer $n_{1}$ such that the star $Y_{2}$ of $p$ in $\mathcal{R}^{n_{1}}\left(Y_{1}\right)$ is contained in the interior of $Y_{1}$. We may replace $Y_{1}$ by $Y_{2}$ and repeat this construction indefinitely. We obtain positive integers $n_{1}, n_{2}, n_{3}, \ldots$ and $\mathcal{R}$-complexes $Y_{1}, Y_{2}, Y_{3}, \ldots$ such that $Y_{i+1} \subseteq \operatorname{int}\left(Y_{i}\right)$ and $Y_{i+1}$ is the star of $p$ in $\mathcal{R}^{n_{i}}\left(Y_{i}\right)$ for every positive integer $i$. Because $\mathcal{R}$ has bounded valence, the complexes $Y_{1}, Y_{2}, Y_{3}, \ldots$ determine only finitely many orientation-preserving isomorphism classes of $\mathcal{R}$-complexes. So there exists a finite $\mathcal{R}$-subcomplex $Y$ of some subdivision of $X$ containing $p$ in its interior and a positive integer $n$ such that the star of $p$ in $\mathcal{R}^{n}(Y)$ is $\mathcal{R}$-isomorphic to $Y$ in an orientation-preserving way. Hence the star of $p$ in $\mathcal{R}^{m n}(Y)$ is $\mathcal{R}$-isomorphic to $Y$ in an orientation-preserving way for all positive integers $m$. Because the mesh of $\mathcal{R}$ approaches 0 , there exists a positive integer $k$ so that $\mathcal{R}^{k}(Y)$ contains an $\mathcal{R}$-subcomplex $S$ which is a closed topological disk with $p$ in its interior. Now we choose a positive integer $m$ so that the star of $p$ in $\mathcal{R}^{m n}(Y)$ is contained in $\operatorname{int}(S)$. Let $N=m n$. Then $S$ is an $\mathcal{R}$-subcomplex of some subdivision of $X$ which is a closed topological disk, $p \in \operatorname{int}(S)$, and there exists an orientation-preserving $\mathcal{R}$ isomorphism from $S$ to a subcomplex $Z$ of $\mathcal{R}^{N}(S)$ with $Z \subseteq \operatorname{int}(S)$. Lemma 2.4 implies that $S$ is $\mathcal{R}$-isomorphic to a seed of an expansion $\mathcal{R}^{N}$-complex.

This proves Lemma 2.5

Remark 2.6. Let $\mathcal{R}$ be a finite subdivision rule with bounded valence and mesh approaching 0. Lemma 2.5 produces expansion complexes, albeit $\mathcal{R}^{N}$-complexes, not $\mathcal{R}$-complexes. However replacing $\mathcal{R}$ by $\mathcal{R}^{N}$ is usually harmless because we are usually interested in whether or not $\mathcal{R}$ is conformal and the bounded overlap theorem [8, Theorem 4.3.1] implies that $\mathcal{R}$ is conformal if and only if $\mathcal{R}^{N}$ is conformal.

Let $\mathcal{R}$ be a finite subdivision rule. Let $X$ be an $\mathcal{R}$-complex. Let $x$ and $y$ be points in $X$. We define the skinny path distance between $x$ and $y$ to be the smallest nonnegative integer $n$ such that there exist tiles $t_{0}, \ldots, t_{n}$ of $X$ for which $x \in t_{0}, y \in t_{n}$ and $t_{i} \cap t_{i+1} \neq \emptyset$ for $i \in\{0, \ldots, n-1\}$. The skinny path distance equips $X$ with a pseudometric $d$, called the skinny path pseudometric. Given $d$, we let $d_{n}$ denote the skinny path pseudometric on $\mathcal{R}^{n}(X)$ for every nonnegative integer $n$. (So $d_{0}=d$.) Note that if $X$ is an expansion $\mathcal{R}$-complex with expansion map $\varphi$, then $d_{n}(x, y)=d\left(\varphi^{n}(x), \varphi^{n}(y)\right)$ for every $x, y \in X$ and every nonnegative integer $n$. Our next lemma gives a basic property of this skinny path pseudometric.

Lemma 2.7. Let $\mathcal{R}$ be a finite subdivision rule whose mesh approaches 0 . Then there exists a positive integer $N$ with the following property. Let $X$ be an $\mathcal{R}$-complex with skinny path pseudometric $d$. Let $x$ and $y$ be points in $X$ such that $d(x, y) \geq 2$. Then $d_{N}(x, y) \geq 2 d(x, y)$. 
Proof. Because the mesh of $\mathcal{R}$ approaches 0 and $\mathcal{R}$ has only finitely many tile types, the following is true.

There exists a positive integer $M$ with the following property. Let $t$ be a tile type of $\mathcal{R}$. Let $e$ be either a vertex or an edge of $t$ and let $f$ be either a vertex or an edge of $t$ such that $e \cap f=\emptyset$. Then no tile of $\mathcal{R}^{M}(t)$ meets both $e$ and $f$.

Now let $x$ and $y$ be points in $X$ such that $d(x, y) \geq 2$. Let $n=d(x, y)$. Given a nonnegative integer $m$, let $B_{x}(m)=\{z \in X: d(x, z) \leq m\}$. Let $m \in\{0, \ldots, n-2\}$. Note that $B_{x}(m)$ and the closure of $X \backslash B_{x}(m+1)$ are disjoint. Hence line 2.8 implies that no tile of $\mathcal{R}^{M}(X)$ meets both $B_{x}(m)$ and the closure of $X \backslash B_{x}(m+1)$. It follows that

$$
d_{M}(x, y) \geq 2(n-1)+1=2 n-1 .
$$

Thus

$$
d_{2 M}(x, y) \geq 2(2 n-1)-1=4 n-3 \geq 2 n=2 d(x, y) .
$$

We may therefore take $N=2 M$, which proves Lemma 2.7

We next prove that expansion maps have unique fixed points.

Lemma 2.9. Let $\mathcal{R}$ be a finite subdivision rule with bounded valence and mesh approaching 0 , and let $X$ be an expansion $\mathcal{R}$-complex with expansion map $\varphi: X \rightarrow$ $X$. Then $\varphi$ has a unique fixed point.

Proof. We can assume without loss of generality that $X=\mathbb{R}^{2}$. For $x, y \in X$, we denote the Euclidean distance between $x$ and $y$ by $\|x-y\|$. Let $d$ denote the skinny path pseudometric on $X$. Let $N$ be as in Lemma 2.7 and let $\tau=\varphi^{-N}$.

Let $x \in X$, and let $Y=\left\{x, \varphi^{-1}(x), \varphi^{-2}(x), \ldots, \varphi^{-N}(x)\right\}$. Let $y \in Y$. Since $d_{N}(\tau(x), \tau(y))=d\left(\varphi^{N}(\tau(x)), \varphi^{N}(\tau(y))\right)=d(x, y)$, Lemma 2.7 implies that if $d(\tau(x), \tau(y)) \geq 2$, then $d(\tau(x), \tau(y)) \leq d_{N}(\tau(x), \tau(y)) / 2=d(x, y) / 2$. Hence there is a positive integer $K$ such that $d\left(\tau^{k}(x), \tau^{k}(y)\right) \leq 1$ for every integer $k \geq K$. Since $Y$ is finite, we may, and do, assume that $K$ is independent of $y$. Taking $y=\varphi^{-N}(x)=\tau(x)$, we see in particular that $d\left(\tau^{K}(x), \tau^{K+1}(x)\right) \leq 1$.

In this paragraph we prove by induction on $k$ that $d\left(\tau^{K}(x), \tau^{k}(y)\right) \leq 2$ for every $y \in Y$ and every integer $k \geq K$. Let $y \in Y$. The previous paragraph handles the case $k=K$. So suppose that $k \geq K$ and that $d\left(\tau^{K}(x), \tau^{k}(y)\right) \leq 2$. If $d\left(\tau^{K+1}(x), \tau^{k+1}(y)\right) \geq 2$, then Lemma 2.7 implies that $d\left(\tau^{K+1}(x), \tau^{k+1}(y)\right) \leq$ $d\left(\tau^{K}(x), \tau^{k}(y)\right) / 2 \leq 1$. Hence $d\left(\tau^{K+1}(x), \tau^{k+1}(y)\right) \leq 1$. By the previous paragraph, $d\left(\tau^{K}(x), \tau^{K+1}(x)\right) \leq 1$. The triangle inequality implies $d\left(\tau^{K}(x), \tau^{k+1}(y)\right) \leq$ 2 , as desired.

The last paragraph shows that there exists a compact subset $C \subseteq X$ which contains all images of $Y$ under the iterates of $\tau$. Let $\epsilon$ be a positive real number. Because the mesh of $\mathcal{R}$ approaches 0 , there exists a positive integer $m$ such that every tile of $\mathcal{R}^{m}(X)$ which meets $C$ has Euclidean diameter less than $\epsilon / 5$. Now apply the argument of the preceding paragraphs to the expansion complex $\mathcal{R}^{m}(X)$ with expansion map $\varphi$. We find that there exists a positive integer $L$ such that $d_{m}\left(\tau^{L}(x), \tau^{k}(y)\right) \leq 2$ for every $y \in Y$ and every integer $k \geq L$. It follows that $\left\|\varphi^{-i}(x), \varphi^{-j}(x)\right\|<\epsilon$ if $i, j \geq L N$, and hence that the sequence $\left\{\varphi^{-j}(x)\right\}$ is a Cauchy sequence. Hence it converges, necessarily to a fixed point of $\varphi$.

We prove by contradiction that the fixed point of $\varphi$ is unique. Suppose that $p$ and $q$ are distinct fixed points of $\varphi$. Let $K=d(p, q)$. Since the mesh of $\mathcal{R}$ 
approaches 0 , there is a positive integer $N$ such that $d_{N}(p, q)>K$. Hence $K=$ $d(p, q)=d\left(\varphi^{N}(p), \varphi^{N}(q)\right)=d_{N}(p, q)>K$. Since this is impossible, the fixed point of $\varphi$ is unique.

This proves Lemma 2.9 .

Let $X$ be a CW complex which is a surface, and let $Y$ be a finite connected CW subcomplex of $X$. By a disk neighborhood of $Y$ we mean a closed topological disk $D$ which is a subcomplex of $X$ with $Y \subseteq \operatorname{int}(D)$. By a minimal disk neighborhood of $Y$ we mean a disk neighborhood of $Y$ which is contained in every disk neighborhood of $Y$.

Lemma 2.10. Let $X$ be a $C W$ complex which is a contractible planar surface, and let $Y$ be a finite connected $C W$ subcomplex of $X$ contained in int $(X)$. Then $Y$ has a minimal disk neighborhood $D$, and $\partial D \subseteq \partial \operatorname{star}(Y, X)$.

Proof. We may, and do, assume that $X \subseteq \mathbb{R}^{2}$. Let $S=\operatorname{star}(Y, X)$. Let $C$ be the unbounded connected component of $\mathbb{R}^{2} \backslash S$. Using the facts that $Y$ is connected and $S$ is a closed neighborhood of $Y$, one checks that $\partial C$ is a simple closed curve. Hence $D=\mathbb{R}^{2} \backslash C$ is a closed topological disk containing $Y$ in its interior. Because $\partial D \subseteq \partial S \subseteq X$ and $X$ is contractible, $D \subseteq X$. Thus $D$ is a disk neighborhood of $Y$, and is clearly the minimal disk neighborhood of $Y$.

We conclude this section with an informal discussion of expansion complexes. For the rest of this section let $\mathcal{R}$ be a bounded valence finite subdivision rule whose mesh approaches 0 . Let $X$ be an expansion $\mathcal{R}$-complex with expansion map $\varphi$ and skinny path pseudometric $d$. Lemma 2.9 shows that $\varphi$ fixes a point $p \in X$.

In this paragraph we show that $\varphi$ really does expand. Let $C$ be a compact subset of $X \backslash\{p\}$, and let $K$ be a positive integer. Because the mesh of $\mathcal{R}$ approaches 0 there exists a nonnegative integer $N$ such that $d_{n}(C, p) \geq K$ for $n \geq N$. Hence $d\left(\varphi^{n}(C), \varphi^{n}(p)\right) \geq K$ for $n \geq N$, and so $d\left(\varphi^{n}(C), p\right) \geq K$ for $n \geq N$. This is what we mean by saying that $\varphi$ really does expand.

In this paragraph we show that every expansion complex has a seed. Let $S$ be the minimal disk neighborhood of $p$. Since $\varphi(p)=p$, it follows that $S \subseteq$ $\varphi(S)$. The previous paragraph shows that if $K$ is a positive integer, then there exists a nonnegative integer $n$ such that $d\left(\varphi^{n}(\partial S), p\right) \geq K$. It follows that $X=$ $\bigcup_{n=0}^{\infty} \varphi^{n}(S)$. Thus $S$ is a seed for $X$. So every expansion complex has a seed.

In this paragraph we show that $X$ has bounded valence. Let $S$ be a seed for $X$. Then every point of $X$ is contained in $\varphi^{n}(S)$ for some nonnegative integer $n$. Hence every point of $X$ is contained in $\varphi^{n}(\operatorname{int}(S))$ for some nonnegative integer $n$. Combining the facts that $S$ is a finite $\mathcal{R}$-complex, $\mathcal{R}$ has bounded valence, and $\varphi^{n}(S)$ is $\mathcal{R}$-isomorphic to $\mathcal{R}^{n}(S)$, we see that $X$ has bounded valence.

\section{Conformal structures}

In this section we introduce partial conformal structures on subdivision complexes. We define partial conformal structures using charts. For convenience, we first triangulate our subdivision complexes in order to simplify the discussion and cut down on the number of overlaps to be considered. We define these triangulations in the next paragraph.

Let $\mathcal{R}$ be a finite subdivision rule. For each tile type $t$, let $\otimes(t)$ be a triangulation of $t$ obtained by adding a barycenter $b(t)$ to $t$ and joining $b(t)$ to each vertex of $t$ 
by an arc. These triangulations push forward under the characteristic maps to give a triangulation $\otimes\left(S_{\mathcal{R}}\right)$ of $S_{\mathcal{R}}$. Next let $X$ be an $\mathcal{R}$-complex with structure map $f: X \rightarrow S_{\mathcal{R}}$. Then the triangulation $\otimes\left(S_{\mathcal{R}}\right)$ pulls back under $f$ to a triangulation $\otimes(X)$ of $X$. We call $\otimes(X)$ the face barycenter triangulation of $X$.

In this paragraph we define butterflies. Let $\mathcal{R}$ be a finite subdivision rule, and let $X$ be an $\mathcal{R}$-complex. A butterfly of $X$ is the union of an open edge $e$ in $X$ together with two distinct open triangles $t_{1}$ and $t_{2}$ in $\otimes(X)$ such that $e$ is contained in the closures of $t_{1}$ and $t_{2}$. Let $b$ be a butterfly of $X$, and let $f: X \rightarrow S_{\mathcal{R}}$ be the structure map of $X$. If $\left.f\right|_{b}$ is injective, then $f(b)$ is a butterfly of $S_{\mathcal{R}}$. In this case we say that $b$ is a nonfolding butterfly. If $\left.f\right|_{b}$ is not injective, then $f$ maps the two open tiles of $b$ to one open tile of $\nabla\left(S_{\mathcal{R}}\right)$. In this case we say that $b$ is a folding butterfly.

For the rest of this section, let $\mathcal{R}$ be a finite subdivision rule and let $X$ be an $\mathcal{R}$ complex. Suppose that there is an orientation on every open tile and every butterfly of $X$. Let $\mathcal{F}$ be the collection of all open tiles and butterflies of $X$. The union of the elements of $\mathcal{F}$ is $\operatorname{int}(X) \backslash V(X)$, where $V(X)$ is the set of vertices of $X$.

In this paragraph we define charts for $X$. For every $s \in \mathcal{F}$ we require that there exist an open set $\widehat{s} \subseteq \mathbb{C}$ and an orientation-preserving homeomorphism $\mu_{s}: s \rightarrow \widehat{s}$, giving us a chart for $s$. Suppose that $s$ is an open tile of $X, t$ is an open triangle of $\nabla(X)$ contained in $s$, and $e$ is the open edge of $X$ contained in the closure of $t$. We also require that $\left.\mu_{s}\right|_{t}$ extends to an injective continuous function from $t \cup e$ into $\mathbb{C}$. (We use this condition to define charts for pullbacks of partial conformal structures over folding butterflies of $\mathcal{R}$-complexes. See two paragraphs below.) We now have an atlas $\mathcal{A}=\left\{\mu_{s}: s \in \mathcal{F}\right\}$ of charts for $X$.

We say that $\mathcal{A}$ is a partial conformal structure on $X$ if these charts satisfy the following compatibility condition. If $s_{1}$ is an open tile in $\mathcal{F}$ and $s_{2}$ is a butterfly in $\mathcal{F}$ such that $s_{1} \cap s_{2} \neq \emptyset$, then the map $\mu_{s_{2}} \circ \mu_{s_{1}}^{-1}: \mu_{s_{1}}\left(s_{1} \cap s_{2}\right) \rightarrow \mu_{s_{2}}\left(s_{1} \cap s_{2}\right)$ is conformal if the orientations of $s_{1}$ and $s_{2}$ agree and is anticonformal if the orientations of $s_{1}$ and $s_{2}$ disagree.

Now let $X$ be an $\mathcal{R}$-complex with structure map $f: X \rightarrow S_{\mathcal{R}}$, and let $\mathcal{A}$ be a partial conformal structure on $S_{\mathcal{R}}$. We pull back the atlas $\mathcal{A}$ to $X$ as follows. Let $\iota: \mathbb{C} \rightarrow \mathbb{C}$ denote complex conjugation. Let $s$ be either an open tile or a nonfolding butterfly of $X$. If $f$ preserves orientation on $s$, then we define the chart for $s$ to be $\mu_{f(s)} \circ f$. If $f$ reverses orientation on $s$, then we define the chart for $s$ to be $\iota \circ \mu_{f(s)} \circ f$. A straightforward verification shows that this defines a partial conformal structure on $X$ if there are no folding butterflies.

We continue the discussion of the previous paragraph, assuming that $X$ is an oriented surface. In this case, the partial conformal structure defined in the previous paragraph is conformal at every point in $\operatorname{int}(X)$ which is neither a vertex nor in an edge whose butterfly is a folding butterfly. Now suppose that $b$ is a folding butterfly of $X$. Then $f(b)=t \cup e$, where $t$ is an open tile of $\nabla\left(S_{\mathcal{R}}\right)$ and $e$ is the open edge of $S_{\mathcal{R}}$ contained in the closure of $t$. Let $s$ be the open tile of $S_{\mathcal{R}}$ which contains $t$. By assumption $\left.\mu_{s}\right|_{t}$ extends to a homeomorphism, denoted by $\mu_{t}$, from $t \cup e$ onto a subset of $\mathbb{C}$. Let $D$ be a closed topological disk in $\{z \in \mathbb{C}: \operatorname{Im}(z) \geq 0\}$ such that $D \cap \mathbb{R}$ is a nontrivial line segment. The Riemann mapping theorem and standard results on boundary behavior (see Section 348 of [9]) imply that there exists an injective continuous map $g: \mu_{t}(t \cup e) \rightarrow D$ which gives a conformal homeomorphism from $\mu_{t}(t)$ to the interior of $D$ such that $g \circ \mu_{t}(e)$ is the interior 
of $D \cap \mathbb{R}$. Now let $t_{1}$ and $t_{2}$ be the open triangles in $b$. Let $i \in\{1,2\}$. If $f$ preserves orientation in mapping $t_{i}$ to $t$, then we set $\mu_{i}=\left.g \circ \mu_{t} \circ f\right|_{t_{i}}$. If $f$ reverses orientation in mapping $t_{i}$ to $t$, then we set $\mu_{i}=\left.\iota \circ g \circ \mu_{t} \circ f\right|_{t_{i}}$. The functions $\mu_{1}$ and $\mu_{2}$ determine a homeomorphism from $b$ to a subset of $\mathbb{C}$, giving a chart for $b$. This chart is compatible with the charts defined above. It is even true that $e$ is an analytic arc in $X$. If we define a chart for $b$ using a closed disk other than $D$ or a function other than $g$, then we obtain another conformal structure on $b$. But the identity map on $b$ is a homeomorphism which maps one conformal structure to the other in the complement of an analytic arc. As on page 183 of [16], it follows that the identity map is conformal. Thus our conformal structure on $b$ is independent of the choice of $D$ and $g$. In this way a partial conformal structure on $S_{\mathcal{R}}$ determines a partial conformal structure on $X$. We need a further condition on the partial conformal structure $\mathcal{A}$ on $S_{\mathcal{R}}$ to ensure that the pullback extends over vertices of $X$ with locally Euclidean neighborhoods. But if $D_{1}$ and $D_{2}$ are open topological disks in $\mathbb{C}$ with $p \in D_{1}$, then a homeomorphism from $D_{1}$ to $D_{2}$ which is conformal in $D_{1} \backslash\{p\}$ is conformal even at $p$. From this it follows that if the conformal structure on $\operatorname{int}(X) \backslash V$ extends to a vertex of $\operatorname{int}(X)$, then that extension is unique.

Let $\mathcal{R}$ be a finite subdivision rule, let $\mathcal{A}$ be a partial conformal structure on $S_{\mathcal{R}}$, and let $v$ be a vertex of $S_{\mathcal{R}}$. Then $v$ is a puncture if whenever $X$ is an $\mathcal{R}$-complex which is an oriented surface and $f: X \rightarrow S_{\mathcal{R}}$ is the structure map such that $p$ is a vertex of $X$ and $f(p)=v$, then the pullback of $\mathcal{A}$ to $X$ has a removable singularity at $p$. The partial conformal structure $\mathcal{A}$ is called nonsingular if every vertex of $S_{\mathcal{R}}$ is a puncture.

We say that a partial conformal structure on $S_{\mathcal{R}}$ is $\mathcal{R}$-invariant or simply invariant if the following holds. Suppose that we have (1) $s \in \mathcal{F},(2) x \in s$, (3) an open neighborhood $U$ of $\mu_{s}(x)$ in $\mu_{s}(s)$ and $(4) s^{\prime} \in \mathcal{F}$ such that $\sigma_{\mathcal{R}}$ maps $\mu_{s}^{-1}(U)$ injectively into $s^{\prime}$. If $\sigma_{\mathcal{R}}$ preserves orientation in mapping $\mu_{s}^{-1}(U)$ to $s^{\prime}$, then we require that $\left.\left(\mu_{s^{\prime}} \circ \sigma_{\mathcal{R}} \circ \mu_{s}^{-1}\right)\right|_{U}$ is conformal. If $\sigma_{\mathcal{R}}$ reverses orientation in mapping $\mu_{s}^{-1}(U)$ to $s^{\prime}$, then we require that $\left.\left(\mu_{s^{\prime}} \circ \sigma_{\mathcal{R}} \circ \mu_{s}^{-1}\right)\right|_{U}$ is anticonformal. Note that this condition is vacuous if either $\sigma_{\mathcal{R}}(x)$ is a vertex of $S_{\mathcal{R}}$ or $x$ lies in the open edge of a folding butterfly $b$ of $\mathcal{R}\left(S_{\mathcal{R}}\right)$ such that $b \cap s$ contains $x$ in its interior.

Suppose that $\mathcal{A}$ is a partial conformal structure on $S_{\mathcal{R}}$. Let $X$ be an oriented surface which is an $\mathcal{R}$-complex. Suppose that $\mathcal{A}$ determines a conformal structure on $\operatorname{int}(X)$. Let $\bar{X}$ denote a uniformization of $X$. The cell structure of $\bar{X}$ is determined only up to a conformal automorphism of the underlying space of $\bar{X}$. If $X$ is homeomorphic to $\mathbb{R}^{2}$, then we take $\bar{X}$ to be either $\mathbb{C}$ or the open unit disk. We say that $X$ is parabolic if $\bar{X}$ is $\mathbb{C}$, and we say that $X$ is hyperbolic if $\bar{X}$ is the open unit disk.

To say that an $\mathcal{R}$-complex $X$ is parabolic, we mean that some partial conformal structure on $S_{\mathcal{R}}$ determines a conformal structure on $X$ for which the uniformization of $X$ is $\mathbb{C}$. Similarly, to say that an $\mathcal{R}$-complex $X$ is hyperbolic, we mean that some partial conformal structure on $S_{\mathcal{R}}$ determines a conformal structure on $X$ for which the uniformization of $X$ is the open unit disk. We will see in Remark 5.8 that if $\mathcal{R}$ has bounded valence with mesh approaching 0 and if $X$ has bounded valence, then the definitions of parabolic and hyperbolic are independent of the conformal structure on $S_{\mathcal{R}}$.

It is not obvious from the definition that every finite subdivision rule has a partial conformal structure. In [7, Section 2] we define a partial conformal structure 
on $S_{\mathcal{R}}$, called the regular conformal structure, for any finite subdivision rule $\mathcal{R}$. This partial conformal structure, which is inspired by a construction given by Bowers and Stephenson in 2], is created by mapping tiles to open regular polygons in $\mathbb{C}$.

\section{Consequences of having a COnformal structure}

Our first result in this section shows the effects that invariant partial conformal structures have on expansion complexes.

Theorem 4.1. Let $\mathcal{R}$ be a finite subdivision rule with bounded valence and mesh approaching 0 , and let $X$ be an oriented expansion $\mathcal{R}$-complex. Then every invariant partial conformal structure on $S_{\mathcal{R}}$ determines a conformal structure on $X$. For

every such conformal structure $X$ is parabolic, and the expansion map of $X$ is conformal.

Proof. Suppose that $S_{\mathcal{R}}$ has an invariant partial conformal structure $\mathcal{A}$. Let $V$ be the set of vertices of $X$. Let $E$ be the set of open edges of $X$ whose butterflies are folding butterflies. As we saw in Section 3 , $\mathcal{A}$ determines a conformal structure on $X \backslash V$ in such a way that every element of $E$ is an analytic arc.

Let $\varphi$ be the expansion map of $X$. Using the definitions, one checks that $\varphi$ is conformal at every point $x \in X$ such that $\varphi(x) \notin V$ and neither $x$ nor $\varphi(x)$ is contained in an element of $E$.

Let $x \in X$ such that $\varphi(x) \in e$ with $e \in E$. Let $U$ be an open neighborhood of $\varphi(x)$ such that the intersection of $U$ with the 1-skeleton of $X$ is $U \cap e$. Then $\varphi^{-1}$ is conformal in $U$ away from the analytic arc $e$. As on page 183 of [16, it follows that $\varphi^{-1}$ is conformal in $U$ and so $\varphi$ is conformal at $x$.

Now let $x$ be a point of $X$ such that $\varphi(x) \notin V$ but $x \in e$ with $e \in E$. Let $U$ be an open neighborhood of $x$ such that $U$ contains no vertex of $\mathcal{R}(X)$ and the intersection of $U$ with the 1-skeleton of $X$ is $U \cap e$. Then $\varphi$ is conformal in $U$ away from the analytic arc $e$. As in the previous paragraph we see that $\varphi$ is conformal at $x$. Hence $\varphi$ is conformal at every point $x \in X$ such that $\varphi(x) \notin V$.

Next let $x \in X \backslash V$ such that $\varphi(x) \in V$. Then there exists a deleted neighborhood $U$ of $x$ which $\varphi$ maps as a conformal homeomorphism to a deleted neighborhood of $\varphi(x)$. Since the conformal structure on $U$ extends to $x$, the conformal structure on $X \backslash V$ extends to $\varphi(x)$ and $\varphi$ is conformal at $x$. It follows inductively that the conformal structure extends over any vertex $v$ such that $\varphi^{-n}(v)$ is not a vertex for some positive integer $n$. Lemma 2.9 and the discussion at the end of Section2 2 show that $\varphi$ has a unique fixed point $p$ and expands. Hence the conformal structure on $X \backslash V$ extends to $X \backslash\{p\}$ and $\varphi$ is conformal on $X \backslash\{p\}$.

So now we have a conformal structure on $X \backslash\{p\}$, and $\varphi$ is a conformal automorphism of $X \backslash\{p\}$. According to Theorem IV.6.1 on page 192 of [1], every Riemann surface with infinite cyclic fundamental group is conformally equivalent to either $\mathbb{C} \backslash\{0\}$, a punctured open disk, or an open ring (annulus). Section V.4 of [11] discusses the conformal automorphisms of these Riemann surfaces. The group of conformal automorphisms of a punctured open disk consists of rotations, and the group of conformal automorphisms of an open ring has a subgroup of index 2 which consists of rotations. Since $\varphi$ is a conformal automorphism of $X \backslash\{p\}$ whose square does not have the dynamics of a rotation, it follows that $X \backslash\{p\}$ is conformally 
equivalent to $\mathbb{C} \backslash\{0\}$. Thus the conformal structure on $X \backslash\{p\}$ extends to $X$ in such a way that $X$ is parabolic and $\varphi$ is conformal.

This proves Theorem 4.1 .

Theorem 4.2. Let $\mathcal{R}$ be a finite subdivision rule with bounded valence and mesh approaching 0 . Then every invariant partial conformal structure on $S_{\mathcal{R}}$ is nonsingular.

Proof. Suppose that $S_{\mathcal{R}}$ has an invariant partial conformal structure $\mathcal{A}$. Let $X$ be an oriented surface which is an $\mathcal{R}$-complex. We must show that the pullback to $X$ of $\mathcal{A}$ determines a conformal structure at every vertex of $X \operatorname{in} \operatorname{int}(X)$. So let $v$ be a vertex of $X$ in $\operatorname{int}(X)$.

Lemma 2.5 implies that there exists an $\mathcal{R}$-subcomplex $S$ of some subdivision of $X$ with $v \in \operatorname{int}(S)$ such that $S$ is $\mathcal{R}$-isomorphic to a seed of some expansion $\mathcal{R}^{N}$-complex $Y$. We orient $Y$. Theorem 4.1 implies that $\mathcal{A}$ determines a conformal structure on $Y$. In particular, $\mathcal{A}$ determines a conformal structure on the subcomplex of $Y$ corresponding to $S$. It follows that the pullback to $X$ of $\mathcal{A}$ extends to $v$.

This proves Theorem 4.2 .

In this paragraph we make a definition to prepare for Lemma 4.3, Let $\mathcal{R}$ be a finite subdivision rule, and let $X$ be an $\mathcal{R}$-complex homeomorphic to $\mathbb{R}^{2}$. Lemma 2.10 implies that every tile of $X$ has a minimal disk neighborhood. We say that $X$ has finite disk neighborhood type if there exist finitely many tiles $t_{1}, \ldots, t_{n}$ of $X$ with minimal disk neighborhoods $D_{1}, \ldots, D_{n}$ having the following property. If $t$ is a tile of $X$ with minimal disk neighborhood $D$, then there exists an integer $m \in\{1, \ldots, n\}$ and an $\mathcal{R}$-isomorphism $f: D_{m} \rightarrow D$ with $f\left(t_{m}\right)=t$. If $X$ has a conformal structure, then we say that $X$ has finite conformal disk neighborhood type if it is furthermore possible to choose $m$ and $f$ so that $f$ is conformal $\operatorname{in} \operatorname{int}\left(D_{m}\right)$.

Bounded valence is not enough to give us the combinatorial finiteness that we need for the proofs of Theorems 4.4, 4.5, and 4.6. We need the stronger condition that there are finitely many disk neighborhood types. This does not follow from the bounded valence property because a closed tile need not be contractible. For example, Figure 8 shows part of an infinite subdivision complex (for a finite subdivision rule such as the fractal hexagonal subdivision rule of [5]) for which no two distinct tiles have the same disk neighborhood type. Lemma 4.3 shows that we can get around this difficulty by subdividing the subdivision complex.

Lemma 4.3. Let $\mathcal{R}$ be a bounded valence finite subdivision rule whose mesh approaches 0 . Let $X$ be a bounded valence $\mathcal{R}$-complex homeomorphic to $\mathbb{R}^{2}$. Then there exists a nonnegative integer $N$ such that the $\mathcal{R}$-complex $\mathcal{R}^{N}(X)$ has finite disk neighborhood type. Moreover, if a partial conformal structure on $S_{\mathcal{R}}$ determines a conformal structure on $X$, then $\mathcal{R}^{N}(X)$ has finite conformal disk neighborhood type.

Proof. We may, and do, assume that $X \subseteq \mathbb{C}$. Since $X$ has bounded valence, there exists a finite collection $T$ of tiles of $X$ such that if $t$ is a tile of $X$, then there exists $s \in T$ and an $\mathcal{R}$-isomorphism from $\operatorname{star}(s, X)$ to $\operatorname{star}(t, X)$ taking $s$ to $t$. Let $s \in T$, and let $S=\operatorname{star}(s, X)$. Then there exists a real number $\epsilon>0$ such that the $2 \epsilon$-neighborhood of $s$ is contained in $S$. Because the mesh of $\mathcal{R}$ approaches 0 , there exists a nonnegative integer $N$ such that the mesh of $\mathcal{R}^{N}(S)$ is less than $\epsilon$. 


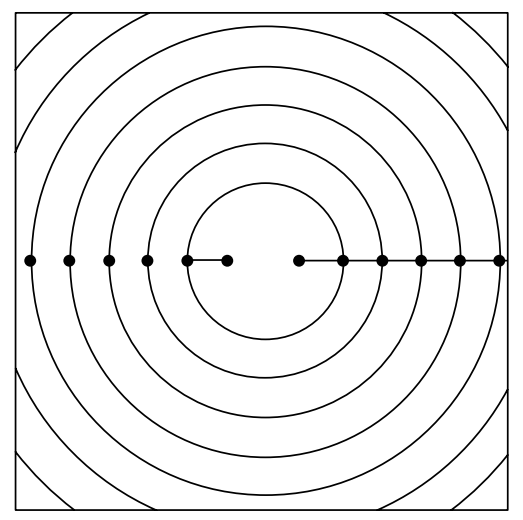

Figure 8. A subdivision complex with infinitely many disk neighborhood types

Let $t$ be a tile of $\mathcal{R}^{N}(s)$, and let $p \in t$. Then the open disk $B$ of radius $2 \epsilon$ centered at $p$ is contained in $S$. Moreover, $\operatorname{star}\left(t, \mathcal{R}^{N}(X)\right) \subseteq B$. Lemma 2.10 implies that $t$ has a minimal disk neighborhood relative to $\mathcal{R}^{N}(X)$ and that it is contained in $B$, hence in $S$. Since $T$ is finite, we may choose $N$ to be independent of $s$.

Now let $t_{1}, \ldots, t_{n}$ be the tiles in $\bigcup_{s \in T} \mathcal{R}^{N}(s)$. Let $D_{1}, \ldots, D_{n}$ be the minimal disk neighborhoods of $t_{1}, \ldots, t_{n}$ relative to $\mathcal{R}^{N}(X)$. Let $t$ be a tile of $\mathcal{R}^{N}(X)$. Let $t^{\prime}$ be the tile of $X$ containing $t$. Then there exists $s \in T$ and an $\mathcal{R}$-isomorphism $f: \operatorname{star}(s, X) \rightarrow \operatorname{star}\left(t^{\prime}, X\right)$ taking $s$ to $t^{\prime}$. It follows that if $D$ is the minimal disk neighborhood of $t$ relative to $\mathcal{R}^{N}(X)$, then there exists an integer $m \in\{1, \ldots, n\}$ such that $f\left(t_{m}\right)=t$ and $f\left(D_{m}\right)=D$. This proves that $\mathcal{R}^{N}(X)$ has finite disk neighborhood type.

Finally, suppose that a partial conformal structure on $S_{\mathcal{R}}$ determines a conformal structure on $X$. Then the function $f$ of the last paragraph is conformal in $\operatorname{int}(\operatorname{star}(s, X))$. Hence $f$ is conformal in $\operatorname{int}\left(D_{m}\right)$. Thus $\mathcal{R}^{N}(X)$ has finite conformal disk neighborhood type.

This proves Lemma 4.3 .

Our next three theorems indicate how a conformal structure affects shapes and sizes of subcomplexes of planar subdivision complexes. We make a definition to prepare for Theorem 4.4. Let $X$ be a subdivision complex with underlying space $\mathbb{R}^{2}$. We say that the tiles of $X$ are almost round if there exists a positive real number $M$ such that if $t$ is a tile of $X$, then there exist concentric circular disks $C$ and $D$ with Euclidean diameters $c$ and $d$, respectively, such that $C \subseteq t \subseteq D$ and $d \leq M c$. If we want to emphasize this constant $M$, we say that the tiles of $X$ are almost round $(M)$.

Theorem 4.4. Let $\mathcal{R}$ be a bounded valence finite subdivision rule whose mesh approaches 0 . Suppose that some partial conformal structure on $S_{\mathcal{R}}$ determines a conformal structure on a bounded valence $\mathcal{R}$-complex which is homeomorphic to $\mathbb{R}^{2}$. Let $X \subseteq \mathbb{C}$ be a uniformization of this $\mathcal{R}$-complex. Then the tiles of $X$ are almost round.

Proof. We begin by verifying that it suffices to prove Theorem 4.4 for some subdivision of $X$. Let $N$ be a nonnegative integer, and suppose that the tiles of $\mathcal{R}^{N}(X)$ 
are almost round $(M)$. There exists a positive integer $K$ such that every tile of $X$ contains at most $K$ tiles of $\mathcal{R}^{N}(X)$. Let $t$ be a tile of $X$. Let $s_{1}, \ldots, s_{k}$ be the tiles of $\mathcal{R}^{N}(t)$. Then there exist concentric circular disks $C_{i}$ and $D_{i}$ with Euclidean diameters $c_{i}$ and $d_{i}$ such that $C_{i} \subseteq s_{i} \subseteq D_{i}$ and $d_{i} \leq M c_{i}$ for $i \in\{1, \ldots, k\}$. Suppose that $d_{i}=\max \left\{d_{1}, \ldots, d_{k}\right\}$. Then $C_{i} \subseteq t$, and the Euclidean diameter of $t$ is at most

$$
\sum_{j=1}^{k} d_{j} \leq k d_{i} \leq K d_{i} \leq K M c_{i} .
$$

It follows that the tiles of $X$ are almost round $(2 K M)$.

We have shown that to prove Theorem 4.4 it suffices to prove it for $\mathcal{R}^{N}(X)$, where $N$ is any nonnegative integer. Lemma 4.3 implies that we may choose $N$ so that $\mathcal{R}^{N}(X)$ has finite conformal disk neighborhood type. We fix such an integer $N$ for the rest of this proof. So $\mathcal{R}^{N}(X)$ has finitely many disk neighborhoods $D_{1}, \ldots, D_{n}$ of tiles $t_{1}, \ldots, t_{n}$ such that if $t$ is a tile of $\mathcal{R}^{N}(X)$, then there exists a disk neighborhood $D$ of $t$, an integer $m \in\{1, \ldots, n\}$, and a cellular isomorphism $f: D_{m} \rightarrow D$ which is conformal in $\operatorname{int}\left(D_{m}\right)$ such that $f\left(t_{m}\right)=t$. For every positive real number $r$ let $B_{r}=\{z \in \mathbb{C}:|z|<r\}$.

For every $m \in\{1, \ldots, n\}$ we apply the Riemann mapping theorem to obtain a conformal homeomorphism $g_{m}: B_{1} \rightarrow \operatorname{int}\left(D_{m}\right)$ with $g_{m}(0) \in \operatorname{int}\left(t_{m}\right)$. Because there are only finitely many of these functions, there exist real numbers $r$ and $R$ with $0<r<R<1$ such that $g_{m}\left(B_{r}\right) \subseteq t_{m} \subseteq g_{m}\left(B_{R}\right)$ for every $m \in\{1, \ldots, n\}$.

Let $t$ be a tile of $\mathcal{R}^{N}(X)$. Then there exists a disk neighborhood $D$ of $t$, an integer $m \in\{1, \ldots, n\}$, and a cellular isomorphism $f: D_{m} \rightarrow D$ which is conformal in $\operatorname{int}\left(D_{m}\right)$ so that $f\left(t_{m}\right)=t$. Let $F$ be the map gotten from $f \circ g_{m}$ by translating $f \circ g_{m}(0)$ to 0 , so that $F(0)=0$, and scaling so that $F^{\prime}(0)=1$. So $F=h \circ f \circ g_{m}$, where $h(z)=a z+b$ for some $a, b \in \mathbb{C}$. Koebe's distortion theorem in line 88 on page 217 of [16] implies that

$$
\frac{r}{(1+r)^{2}} \leq|F(z)| \quad \text { if }|z|=r
$$

and

$$
|F(z)| \leq \frac{R}{(1-R)^{2}} \quad \text { if }|z|=R
$$

This means that $h(t)$ contains the closed disk of radius $r /(1+r)^{2}$ centered at 0 and $h(t)$ is contained in the closed disk of radius $R /(1-R)^{2}$ centered at 0 . Since $r$ and $R$ are independent of $t$, it follows that the tiles of $\mathcal{R}^{N}(X)$ are almost round.

This proves Theorem 4.4 .

Theorem 4.5. Let $\mathcal{R}$ be a bounded valence finite subdivision rule whose mesh approaches 0. Suppose that some partial conformal structure on $S_{\mathcal{R}}$ determines a conformal structure on a bounded valence $\mathcal{R}$-complex which is homeomorphic to $\mathbb{R}^{2}$. Let $X \subseteq \mathbb{C}$ be a uniformization of this $\mathcal{R}$-complex. Then for every $p \in \mathbb{C}$ there exists a positive real number $c$, which depends on $p$, with the following property. If $t$ is a tile of $X$ with Euclidean diameter $\delta$ such that the Euclidean distance from $t$ to $p$ is $d>0$, then $\delta \leq c d$.

Proof. We proceed much as in the proof of Theorem 4.4. We begin by verifying that it suffices to prove Theorem 4.5 for some subdivision of $X$. Let $N$ be a nonnegative integer, let $p \in \mathbb{C}$, and suppose that $c$ is a positive real number as in Theorem 4.5 
relative to $\mathcal{R}^{N}(X)$. There exists a positive integer $K$ such that every tile of $X$ contains at most $K$ tiles of $\mathcal{R}^{N}(X)$. Let $t$ be a tile of $X$ with $p \notin t$. Let $s$ be a tile of $\mathcal{R}^{N}(t)$ such that the Euclidean distance $d$ from $s$ to $p$ equals the Euclidean distance from $t$ to $p$. Let $\delta$ be the Euclidean diameter of $s$. We have $\delta \leq c d$. So the maximum Euclidean distance from a point of $s$ to $p$ is at most $(1+c) d$. One sees inductively that the maximum Euclidean distance from a point of $t$ to $p$ is at most $(1+c)^{K} d$. So the Euclidean diameter of every tile of $\mathcal{R}^{N}(t)$ is at most $c(1+c)^{K} d$. So the Euclidean diameter of $t$ is at most $K c(1+c)^{K} d$. It follows that Theorem 4.5 holds for $X$ with $c$ replaced by $K c(1+c)^{K}$.

We have shown that to prove Theorem 4.5] it suffices to prove it for $\mathcal{R}^{N}(X)$, where $N$ is any nonnegative integer. Lemma 4.3 implies that we may choose $N$ so that $\mathcal{R}^{N}(X)$ has finite conformal disk neighborhood type. We fix such an integer $N$ for the rest of this proof. So $\mathcal{R}^{N}(X)$ has finitely many disk neighborhoods $D_{1}, \ldots, D_{n}$ of tiles $t_{1}, \ldots, t_{n}$ such that if $t$ is a tile of $\mathcal{R}^{N}(X)$, then there exists a disk neighborhood $D$ of $t$, an integer $m \in\{1, \ldots, n\}$, and a cellular isomorphism $f: D_{m} \rightarrow D$ which is conformal in $\operatorname{int}\left(D_{m}\right)$ such that $f\left(t_{m}\right)=t$. For every positive real number $r$ let $B_{r}=\{z \in \mathbb{C}:|z|<r\}$.

For every $m \in\{1, \ldots, n\}$ and every $q \in t_{m}$ we apply the Riemann mapping theorem to obtain a conformal homeomorphism $g_{m, q}: B_{1} \rightarrow \operatorname{int}\left(D_{m}\right)$ with $g_{m, q}(0)=q$. For a fixed $m$ and varying $q$, the hyperbolic diameters of the sets $g_{m, q}^{-1}\left(t_{m}\right)$ are equal and these sets all contain 0 . So there exists a real number $R$ with $0<R<1$ such that $t_{m} \subseteq g_{m, q}\left(B_{R}\right)$ for every $m \in\{1, \ldots, n\}$ and every $q \in t_{m}$.

Let $p \in \mathbb{C}$, and let $t$ be a tile of $\mathcal{R}^{N}(X)$. Let $q^{\prime}$ be a point of $t$ whose Euclidean distance from $p$ is minimal. Then there exists a disk neighborhood $D$ of $t$, an integer $m \in\{1, \ldots, n\}$, and a cellular isomorphism $f: D_{m} \rightarrow D$ which is conformal $\operatorname{in} \operatorname{int}\left(D_{m}\right)$ so that $f\left(t_{m}\right)=t$. Assume that $p \notin D$. Let $q=f^{-1}\left(q^{\prime}\right)$. Let $F$ be the map gotten from $f \circ g_{m, q}$ by translating $q^{\prime}$ to 0 , so that $F(0)=0$, and scaling so that $F^{\prime}(0)=1$. So $F=h \circ f \circ g_{m, q}$, where $h(z)=a z+b$ for some $a, b \in \mathbb{C}$. Now Koebe's $\frac{1}{4}$-theorem [16, page 214] implies that $|h(p)| \geq \frac{1}{4}$, and Koebe's distortion theorem in line 88 on page 217 of [16] gives

$$
|F(z)| \leq \frac{R}{(1-R)^{2}} \quad \text { if }|z|=R
$$

So the Euclidean distance from $h(t)$ to $h(p)$ is at least $1 / 4$ and the Euclidean diameter of $h(t)$ is at most $2 R /(1-R)^{2}$. Hence $\delta / d \leq 8 R /(1-R)^{2}$.

We have just proved Theorem 4.5 for the case in which $p \notin D$. Because there are only finitely many cellular isomorphism types of disk neighborhoods such as $D$ and each one is a finite CW complex, we have just proved Theorem 4.5 for all but finitely many tiles $t$ of $\mathcal{R}^{N}(X)$.

Theorem 4.5 follows easily.

We need the following definitions for Theorem 4.6. If $A$ is a bounded subset of $\mathbb{R}^{2}$ with at least two points, then there exists a unique closed metric disk $D \subseteq \mathbb{R}^{2}$ which contains $A$ of minimal Euclidean diameter: $D \subseteq \mathbb{R}^{2}$ is a closed metric disk, $A \subseteq D$, and if $D^{\prime} \neq D$ is a closed metric disk in $\mathbb{R}^{2}$ such that $A \subseteq D^{\prime}$, then the Euclidean diameter of $D$ is less than the Euclidean diameter of $D^{\prime}$. We call $D$ the circumscribing disk of $A$.

Now let $X$ be a subdivision complex contained in $\mathbb{R}^{2}$. Given a subcomplex $Y$ of $X$ and a nonnegative integer $K$, we let $\operatorname{star}^{K}(Y, X)$ denote the $K$-fold star of 
$Y$ in $X$. In other words, $\operatorname{star}^{0}(Y, X)=Y$ and if $K>0$, then $\operatorname{star}^{K}(Y, X)$ is the subcomplex of $X$ whose points have distance at most $K-1$ from $Y$ in the skinny path pseudometric on $X$. If $t$ is a tile of $X$ and if $K$ is a nonnegative integer, then we let $\delta_{K}(t)$ be the Euclidean diameter of the circumscribing disk of $\operatorname{star}^{K}(t, X)$.

Theorem 4.6. Let $\mathcal{R}$ be a bounded valence finite subdivision rule whose mesh approaches 0 . Suppose that some partial conformal structure on $S_{\mathcal{R}}$ determines a conformal structure on an expansion $\mathcal{R}$-complex. Let $X \subseteq \mathbb{C}$ be a uniformization of this expansion $\mathcal{R}$-complex. Let $K$ be a nonnegative integer. Then there exists a real number $c$ with $0<c<1$ such that if $t$ is a tile of $X$, then $\delta_{K}(t) \leq c \delta_{K+1}(t)$.

Proof. Let $\varphi$ be the expansion map of $X$. Lemma 4.3 implies that there exists a nonnegative integer $M$ such that $\mathcal{R}^{M}(X)$ has finite disk neighborhood type. Since $\varphi^{M}: \mathcal{R}^{M}(X) \rightarrow X$ is an $\mathcal{R}$-isomorphism, $X$ has finite disk neighborhood type.

Because the mesh of $\mathcal{R}$ approaches 0 , there exists a nonnegative integer $N$ with the following property. Let $s$ be a tile of $\mathcal{R}^{N}(X)$, let $t$ be the tile of $X$ containing $s$, and let $D$ be the minimal disk neighborhood of $t$ relative to $X$. Then $\operatorname{star}^{K}\left(s, \mathcal{R}^{N}(X)\right) \subseteq \operatorname{int}\left(\mathcal{R}^{N}(D)\right)$. Hence $\operatorname{star}^{K}\left(\varphi^{N}(s), X\right) \subseteq \operatorname{int}\left(\varphi^{N}(D)\right)$. This means that $\varphi^{N}(D)$ is a disk neighborhood of $\operatorname{star}^{K}\left(\varphi^{N}(s), X\right)$. Every tile of $X$ has the form $\varphi^{N}(s)$ for some tile $s$ of $\mathcal{R}^{N}(X)$. Furthermore, because $X$ has finite disk neighborhood type, there are only finitely many $\mathcal{R}$-isomorphism classes of $\mathcal{R}$-complexes of the form $\varphi^{N}(D)$. Thus there exist finitely many tiles $t_{1}, \ldots, t_{n}$ of $X$ and minimal disk neighborhoods $D_{1}, \ldots, D_{n}$ of $\operatorname{star}^{K}\left(t_{1}, X\right), \ldots, \operatorname{star}^{K}\left(t_{n}, X\right)$ having the following property. If $t$ is a tile of $X$ and if $D$ is the minimal disk neighborhood of $\operatorname{star}^{K}(t, X)$, then there exist an integer $m \in\{1, \ldots, n\}$ and an $\mathcal{R}$-isomorphism $f: D_{m} \rightarrow D$ with $f\left(\operatorname{star}^{K}\left(t_{m}, X\right)\right)=\operatorname{star}^{K}(t, X)$. The conformal structure on $X$ is defined so that $f$ is necessarily conformal in $\operatorname{int}\left(D_{m}\right)$. Let $B$ be the open unit disk in $\mathbb{C}$. For every $m \in\{1, \ldots, n\}$ we apply the Riemann mapping theorem to obtain a conformal homeomorphism $g_{m}: B \rightarrow \operatorname{int}\left(D_{m}\right)$.

Now let $t$ be a tile of $X$. Let $D$ be the minimal disk neighborhood of $\operatorname{star}^{K}(t, X)$. The previous paragraph shows that there exists an integer $m \in\{1, \ldots, n\}$ and an $\mathcal{R}$-isomorphism $f: D_{m} \rightarrow D$ such that $f\left(\operatorname{star}^{K}\left(t_{m}, X\right)\right)=\operatorname{star}^{K}(t, X)$ and $f$ is conformal in $\operatorname{int}\left(D_{m}\right)$. Let $h$ be a linear polynomial over $\mathbb{C}$ which maps the interior of the circumscribing disk of $\operatorname{star}^{K+1}(t, X)$ bijectively to $B$. Lemma 2.10 implies that $h(D) \subseteq B$. The function $h \circ f \circ g_{m}: B \rightarrow B$ is conformal, and so as in Theorem 3 of Section 1 of Chapter VIII of [13, it does not increase hyperbolic distance. Because

$$
g_{m}^{-1} \circ f^{-1}\left(\operatorname{star}^{K}(t, X)\right)=g_{m}^{-1}\left(\operatorname{star}^{K}\left(t_{m}, X\right)\right),
$$

there exists a bound independent of $t$ on the hyperbolic diameter of the circumscribing disk of $g_{m}^{-1} \circ f^{-1}\left(\operatorname{star}^{K}(t, X)\right)$. Since $h \circ f \circ g_{m}$ does not increase hyperbolic distance, there exists a bound independent of $t$ on the hyperbolic diameter of the circumscribing disk of $h\left(\operatorname{star}^{K}(t, X)\right)$. Hence there exists a real number $c$ independent of $t$ with $0<c<1$ such that the Euclidean diameter of the circumscribing disk of $h\left(\operatorname{star}^{K}(t, X)\right)$ is less than $2 c$. Since $h$ is a linear polynomial over $\mathbb{C}$, it follows that $\delta_{K}(t) \leq c \delta_{K+1}(t)$.

This proves Theorem 4.6 .

With Theorems 4.4, 4.5, and 4.6 in hand, we have some control of the shapes and sizes of tiles in planar subdivision complexes. The next result involves the 
first application of these theorems. We refer the reader to Section 1.2 of [5] for an introduction to (combinatorial) conformality for finite subdivision rules. Given a subdivision complex $X$, let $\mathcal{S}(X)$ denote the set of tiles of $X$. The "S" stands for shingling, although this shingling is in fact a tiling.

Theorem 4.7. Let $\mathcal{R}$ be a finite subdivision rule with bounded valence and mesh approaching 0 . Suppose that $\mathcal{R}$ has an invariant partial conformal structure. Then $\mathcal{R}$ is conformal.

Proof. In [5] we defined a finite subdivision rule $\mathcal{R}$ to be conformal if $(Y, \mathcal{R})$ is conformal for every surface $Y$ which has the structure of an $\mathcal{R}$-complex. To say that $(Y, \mathcal{R})$ is conformal means that Axiom I and Axiom II of 3 hold in some compact neighborhood of every point in $\operatorname{int}(Y)$ for the sequence of tilings $\left\{\mathcal{S}\left(\mathcal{R}^{n}(Y)\right)\right\}$. We don't verify the axioms directly. By [3, Theorem 7.1], it suffices to prove that every point $p$ of such a subdivision complex $Y$ has a compact neighborhood that is $\mathcal{R}$ isomorphic to a planar $\mathcal{R}$-complex $X_{p}$ whose sequence of tilings $\left\{\mathcal{S}\left(\mathcal{R}^{n}\left(X_{p}\right)\right)\right\}$ is uniformly almost round.

So let $Y$ be a surface which has the structure of an $\mathcal{R}$-complex, and let $p \in \operatorname{int}(Y)$. Lemma 2.5 implies that there exists an $\mathcal{R}$-subcomplex $S$ of some subdivision of $Y$ with $p \in \operatorname{int}(S)$ such that $S$ is $\mathcal{R}$-isomorphic to a seed of some expansion $\mathcal{R}^{N}$ complex $X$. We orient $X$. Theorem 4.1] applied to the finite subdivision rule $\mathcal{R}^{N}(X)$, implies that the invariant partial conformal structure on $S_{\mathcal{R}}$ determines a conformal structure on $X$, that $X$ is parabolic, and that the expansion map of $X$ is conformal. Let $\bar{X}$ be a uniformization of $X$, and let $\varphi$ be the expansion map of $\bar{X}$.

Since $\bar{X}$ has bounded valence, Theorem 4.4 implies that the tiles of $\bar{X}$ are almost round. Since $\varphi$ is a conformal automorphism of $\mathbb{C}$ and $\varphi^{n}: \mathcal{R}^{n N}(\bar{X}) \rightarrow \bar{X}$ is an $\mathcal{R}^{N}$-isomorphism for every nonnegative integer $n$, it follows that the sequence of tilings $\left\{\mathcal{S}\left(\mathcal{R}^{n N}(\bar{X})\right)\right\}$ is uniformly almost round. In other words, there exists a positive real number $M$ such that the tiles of $\mathcal{R}^{n N}(\bar{X})$ are almost round $(M)$ for every nonnegative integer $n$. Now [3, Theorem 7.1] implies that the sequence $\left\{\mathcal{S}\left(\mathcal{R}^{n N}(\bar{X})\right)\right\}$ is conformal in $\bar{X}$. In particular, it is conformal in the subcomplex of $\bar{X}$ corresponding to $S$. Therefore the sequence of tilings $\left\{\mathcal{S}\left(\mathcal{R}^{n N}(Y)\right)\right\}$ is conformal in $S$. As in Remark [2.6, the bounded overlap theorem [8, Theorem 4.3.1] now implies that the sequence of tilings $\left\{\mathcal{S}\left(\mathcal{R}^{n}(Y)\right)\right\}$ is conformal in $S$.

This proves Theorem 4.7

\section{Modulus estimates}

This section deals with combinatorial moduli. We refer the reader to Section 1.2 of [5] for an introduction to combinatorial moduli in the setting of finite subdivision rules.

In this paragraph we note a basic property of expansion maps related to combinatorial moduli. Recall that if $X$ is a subdivision complex, then we let $\mathcal{S}(X)$ denote the shingling of $X$ whose elements are the tiles of $X$. Let $\mathcal{R}$ be a finite subdivision rule, and let $X$ be an expansion $\mathcal{R}$-complex with expansion map $\varphi$. Let $Y$ be either a quadrilateral or a ring in $X$. Then because $\varphi: \mathcal{R}(X) \rightarrow X$ is an $\mathcal{R}$-isomorphism, $M(Y, \mathcal{S}(\mathcal{R}(X)))=M(\varphi(Y), \mathcal{S}(X))$, where $M$ denotes the fat flow modulus. More generally, $M\left(Y, \mathcal{S}\left(\mathcal{R}^{n}(X)\right)\right)=M\left(\varphi^{n}(Y), \mathcal{S}(X)\right)$ for every nonnegative integer $n$. Of course, the corresponding equality also holds for fat cut moduli. In this paper 
we work only with fat flow moduli and fat cut moduli, not skinny flow moduli or skinny cut moduli.

Our first result in this section is the following preparatory lemma.

Lemma 5.1. Let $R$ be a ring in $\mathbb{R}^{2}$. Let $\mathcal{T}$ be an almost round $(M)$ tiling of a subset of $\mathbb{R}^{2}$ which contains $R$. Let $D$ denote the Euclidean diameter of the inner boundary component of $R$, and let $H$ denote the Euclidean height of $R$. Let $A$ denote the Lebesgue area of the union of the tiles $t$ in $\mathcal{T}$ such that $t \cap R$ has nonzero Lebesgue area. Then

$$
m(R, \mathcal{T}) \leq \frac{4 M^{2} A}{\pi D^{2}} \quad \text { and } \quad \frac{\pi H^{2}}{4 M^{2} A} \leq M(R, \mathcal{T}) .
$$

Proof. We define a weight function $\rho$ on $\mathcal{T}$ as follows. Let $t \in \mathcal{T}$. Because $\mathcal{T}$ is almost round $(M)$, there exist concentric circular disks $C(t)$ and $D(t)$ with Euclidean diameters $c(t)$ and $d(t)$, respectively, such that $C(t) \subseteq t \subseteq D(t)$ and $d(t) \leq M c(t)$. If the Lebesgue area of $t \cap R$ is 0 , then we set $\rho(t)=0$. If the Lebesgue area of $t \cap R$ is not 0 , then we set $\rho(t)=d(t)$. This defines $\rho$.

Let $C$ be the inner boundary component of $R$. Since $C$ is compact, there exist points $x_{1}, x_{2} \in C$ such that the distance between $x_{1}$ and $x_{2}$ is $D$. Let $L$ be the line in $\mathbb{R}^{2}$ containing $x_{1}$ and $x_{2}$. Let $R_{1}$ and $R_{2}$ be the rays in $L$ based at $x_{1}$ and $x_{2}$, respectively, such that $R_{1} \cap R_{2}=\emptyset$. Let $\alpha$ be a simple closed curve in $R$ separating the ends of $R$. Then $\alpha$ meets both $R_{1}$ and $R_{2}$. It follows that there exist distinct tiles $t_{1}, \ldots, t_{k} \in \mathcal{T}$ with nonzero $\rho$-weights which meet $\alpha$ such that $t_{1} \cap R_{1} \neq \emptyset$, $t_{i} \cap t_{i+1} \neq \emptyset$ for $i \in\{1, \ldots, k-1\}$ and $t_{k} \cap R_{2} \neq \emptyset$. From this it easily follows that $C(R, \rho) \geq D$. Similarly, $H(R, \rho) \geq H$. Moreover, if $t_{1}, \ldots, t_{p}$ are the tiles of $\mathcal{T}$ with nonzero $\rho$-weights, then

$$
A(R, \rho)=\sum_{i=1}^{p} d\left(t_{i}\right)^{2} \leq M^{2} \sum_{i=1}^{p} c\left(t_{i}\right)^{2} \leq \frac{4}{\pi} M^{2} A .
$$

Thus

$$
m(R, \mathcal{T}) \leq m(R, \rho)=\frac{A(R, \rho)}{C(R, \rho)^{2}} \leq \frac{4 M^{2} A}{\pi D^{2}}
$$

and

$$
M(R, \mathcal{T}) \geq M(R, \rho)=\frac{H(R, \rho)^{2}}{A(R, \rho)} \geq \frac{\pi H^{2}}{4 M^{2} A} .
$$

This proves Lemma 5.1 .

Intuitively, combinatorial moduli of large rings in expansion complexes with almost round tiles should be roughly the same as their classical moduli. To a large extent this section consists of a collection of limited results of this sort. Theorem 5.2 and Theorem 5.4 form a pair of such results and Theorem 5.5] and Theorem 5.6 form another pair of such results. Taken together, they give combinatorial criteria for determining whether an expansion complex is hyperbolic or parabolic.

Theorem 5.2. Let $\mathcal{R}$ be a bounded valence finite subdivision rule whose mesh approaches 0 , and let $X$ be an expansion $\mathcal{R}$-complex in $\mathbb{R}^{2}$ with expansion map $\varphi$. Suppose that there exists an almost round tiling of $X$ which has bounded overlap with $\mathcal{S}(X)$. Then there exists a positive real number $K$ with the following property. 
If $S$ is a ring in $X$ and $R$ is a ring in the interior of $S$ which separates the ends of $S$, then

$$
m\left(R, \mathcal{S}\left(\mathcal{R}^{n}(X)\right)\right) \leq K \frac{A\left(\varphi^{n}(S)\right)}{D\left(\varphi^{n}(S)\right)^{2}}
$$

for every nonnegative integer $n$, where $A\left(\varphi^{n}(S)\right)$ denotes the Lebesgue area of $\varphi^{n}(S)$ and $D\left(\varphi^{n}(S)\right)$ denotes the Euclidean diameter of the inner boundary component of $\varphi^{n}(S)$.

Proof. Let $S$ be a ring in $X$, and let $R$ be a ring in the interior of $S$ which separates the ends of $S$. According to the discussion at the beginning of this section, $m\left(R, \mathcal{S}\left(\mathcal{R}^{n}(X)\right)\right)=m\left(\varphi^{n}(R), \mathcal{S}(X)\right)$ for every nonnegative integer $n$. We estimate the latter modulus. Let $\mathcal{T}$ be an almost round $(M)$ tiling of $X$ which has bounded overlap with $\mathcal{S}(X)$. The bounded overlap theorem [8, Theorem 4.3.1] implies that it suffices to estimate $m\left(\varphi^{n}(R), \mathcal{T}\right)$ instead of $m\left(\varphi^{n}(R), \mathcal{S}(X)\right)$. Because the mesh of $\mathcal{R}$ approaches 0 , given a positive integer $q$ there exists a positive integer $N$ such that $\operatorname{star}^{q}\left(R, \mathcal{S}\left(\mathcal{R}^{n}(X)\right)\right) \subseteq S$ for $n \geq N$, hence $\operatorname{star}^{q}\left(\varphi^{n}(R), \mathcal{S}(X)\right) \subseteq \varphi^{n}(S)$ for $n \geq N$. Because $\mathcal{T}$ and $\mathcal{S}(X)$ have bounded overlap, it is therefore possible to choose $N$ so large that $\operatorname{star}\left(\varphi^{n}(R), \mathcal{T}\right) \subseteq \varphi^{n}(S)$ for every integer $n \geq N$. Let $n$ be an integer with $n \geq N$.

Lemma 5.1 implies that

$$
m\left(\varphi^{n}(R), \mathcal{T}\right) \leq \frac{4 M^{2} A}{\pi D^{2}},
$$

where $A$ is the Lebesgue area of the union of the tiles $t$ in $\mathcal{T}$ for which the Lebesgue area of $t \cap \varphi^{n}(R)$ is not 0 and $D$ is the Euclidean diameter of the inner boundary component of $\varphi^{n}(R)$. Since $\operatorname{star}\left(\varphi^{n}(R), \mathcal{T}\right) \subseteq \varphi^{n}(S)$, we have $A \leq A\left(\varphi^{n}(S)\right)$. Clearly $D \geq D\left(\varphi^{n}(S)\right)$. Hence

$$
m\left(\varphi^{n}(R), \mathcal{T}\right) \leq \frac{4 M^{2}}{\pi} \frac{A\left(\varphi^{n}(S)\right)}{D\left(\varphi^{n}(S)\right)^{2}} .
$$

This provides a bound of the desired sort for $n \geq N$. We finish by increasing the bound if necessary to handle the values of $n$ less than $N$.

This proves Theorem 5.2 ,

Corollary 5.3. Let $\mathcal{R}$ be a bounded valence finite subdivision rule whose mesh approaches 0 . If $X$ is a hyperbolic expansion $\mathcal{R}^{N}$-complex, then $(X, \mathcal{R})$ is not conformal. Hence $\mathcal{R}$ is not conformal.

Proof. Let $X$ be a hyperbolic expansion $\mathcal{R}^{N}$-complex. To prove that $(X, \mathcal{R})$ is not conformal it suffices to prove that $\left(X, \mathcal{R}^{N}\right)$ is not conformal. So we assume without loss of generality that $X$ is an expansion $\mathcal{R}$-complex. We may furthermore replace $X$ by its uniformization. So $X$ is the open unit disk. Since $X$ has bounded valence, Theorem 4.4 implies that the tiles of $X$ are almost round. Hence the assumptions of Theorem 5.2 are satisfied. Let $S$ be a ring in $X$ surrounding the fixed point of the expansion map $\varphi$. Let $R$ be a ring in the interior of $S$ which separates the ends of $S$. Then the areas $A\left(\varphi^{n}(S)\right)$ converge to 0 , but the Euclidean diameters $D\left(\varphi^{n}(S)\right)$ are bounded from 0 . Theorem 5.2 implies that the moduli $m\left(R, \mathcal{S}\left(\mathcal{R}^{n}(X)\right)\right)$ degenerate to 0 . Thus $(X, \mathcal{R})$ is not conformal, and so $\mathcal{R}$ is not conformal.

Theorem 5.4. Let $\mathcal{R}$ be a bounded valence finite subdivision rule whose mesh approaches 0 . Let $X$ be an expansion $\mathcal{R}$-complex in $\mathbb{R}^{2}$ with expansion map $\varphi$ such 
that the tiles of $X$ are almost round. Then there exists a positive real number $K$ with the following property. If $R$ is a ring in $X$ which is a union of tiles of $X$, then

$$
K \frac{H\left(\varphi^{n}(R)\right)^{2}}{A\left(\varphi^{n}(R)\right)} \leq M\left(R, \mathcal{S}\left(\mathcal{R}^{n}(X)\right)\right)
$$

for every nonnegative integer $n$, where $A\left(\varphi^{n}(R)\right)$ denotes the Lebesgue area of $\varphi^{n}(R)$ and $H\left(\varphi^{n}(R)\right)$ denotes the Euclidean height of $\varphi^{n}(R)$.

Proof. Let $n$ be a nonnegative integer. According to the discussion at the beginning of this section, $M\left(R, \mathcal{S}\left(\mathcal{R}^{n}(X)\right)\right)=M\left(\varphi^{n}(R), \mathcal{S}(X)\right)$. We estimate the latter modulus.

The tiling $\mathcal{S}(X)$ is almost round $(M)$ for some positive real number $M$. Hence Lemma 5.1 implies that

$$
M\left(\varphi^{n}(R), \mathcal{S}(X)\right) \geq \frac{\pi}{4 M^{2}} \frac{H\left(\varphi^{n}(R)\right)^{2}}{A\left(\varphi^{n}(R)\right)} .
$$

This proves Theorem 5.4 .

We next show that large rings have large combinatorial moduli in bounded valence parabolic subdivision complexes.

Theorem 5.5. Let $\mathcal{R}$ be a bounded valence finite subdivision rule whose mesh approaches 0 . Let $X$ be a bounded valence parabolic $\mathcal{R}$-complex. Let $R_{1}, R_{2}, R_{3}, \ldots$ be rings in $X$ whose inner boundary components are contained in a fixed compact set such that for every compact subset $C$ of $X$ there is a positive integer I for which the outer boundary component of $R_{i}$ encircles $C$ for every integer $i \geq I$. Then $\lim _{i \rightarrow \infty} M\left(R_{i}, \mathcal{S}(X)\right)=\infty$.

Proof. To prove Theorem 5.5 we may replace $X$ by its uniformization, so we assume that the underlying space of $X$ is $\mathbb{C}$. Theorem 4.4 implies that the tiles of $X$ are almost round $(M)$ for some positive real number $M$. We may choose coordinates in the plane so that the inner boundary components of $R_{1}, R_{2}, R_{3}, \ldots$ are encircled by the unit circle. We employ the tin can argument, which appears in the proof of [3. Proposition 4.0.3] as well as in Sections 3 and 5 of [4. For every real number $r$ set

$$
D_{r}=\left\{z \in \mathbb{C}:|z| \leq e^{r}\right\} .
$$

In this paragraph we show that there exists a positive integer $L$ with the following property. If $t$ is a tile of $X$ and $r$ is a nonnegative real number such that $t \cap D_{r} \neq \emptyset$, then $t \subseteq D_{r+L}$. To begin the proof of this, we note that Theorem 4.5 implies that there exists a positive real number $c$ with the following property. If $t$ is a tile of $X$ with Euclidean diameter $\delta$ such that the Euclidean distance from $t$ to 0 is $d>0$, then $\delta \leq c d$. We choose $L$ to be a positive integer such that $e^{L}$ is greater than the Euclidean diameter of every tile of $X$ which contains 0 and $1+c \leq e^{L}$. Now let $t$ be a tile of $X$, and let $r$ be a nonnegative real number such that $t \cap D_{r} \neq \emptyset$. If $0 \in t$, then $t \subseteq D_{L} \subseteq D_{r+L}$. If $0 \notin t$, then the Euclidean diameter of $t$ is at most $c e^{r}$, and so the distance from 0 to every point of $t$ is at most $(1+c) e^{r} \leq e^{L} \cdot e^{r}=e^{r+L}$. Hence $t \subseteq D_{r+L}$. Thus $L$ has the desired property.

Now let $N$ be a positive integer. By assumption there exists a positive integer $I$ such that the outer boundary component of $R_{i}$ encircles $D_{N}$ for every integer $i \geq I$. We fix $I$ and an integer $i \geq I$. For every $n \in\{1, \ldots, N\}$ set

$$
C_{n}=\left\{t \in \mathcal{S}(X): t \cap D_{n-1}=\emptyset \text { but } t \cap D_{n} \neq \emptyset\right\} .
$$


Let $C_{0}=\left\{t \in \mathcal{S}(X): t \cap D_{0} \neq \emptyset\right\}$, and let $C_{\infty}=\mathcal{S}(X) \backslash\left(C_{0} \cup \cdots \cup C_{N}\right)$. Then $\mathcal{S}(X)$ is the disjoint union of $C_{0}, C_{1}, \ldots, C_{N}, C_{\infty}$. Define a weight function $\rho$ on $\mathcal{S}(X)$ so that if $t$ is a tile of $\mathcal{S}(X)$, then $\rho(t)$ is the Euclidean diameter of $t$. Define another weight function $\rho^{\prime}$ on $\mathcal{S}(X)$ so that if $t \in C_{\infty}$, then $\rho^{\prime}(t)=0$, and if $t \in C_{n}$ with $n \in\{0, \ldots, N\}$, then

$$
\rho^{\prime}(t)=\rho(t) \frac{e^{1-n}}{e-1}
$$

In this paragraph we estimate the $\rho^{\prime}$-height $H\left(R_{i}, \rho^{\prime}\right)$ of $R_{i}$. Let $\gamma$ be a curve in $R_{i}$ joining the ends of $R_{i}$. Then for every $n \in\{1, \ldots, N\}$ there exists an open subcurve $\gamma_{n}$ of $\gamma$ irreducibly joining $D_{n-1}$ and $\mathbb{C} \backslash D_{n}$. The second paragraph of this proof implies that no tile of $X$ meets more than $L+1$ of the curves $\gamma_{1}, \ldots, \gamma_{N}$. So the $\rho^{\prime}$-length of $\gamma$ is at least $1 /(L+1)$ times the sum of the $\rho^{\prime}$-lengths of $\gamma_{1}, \ldots, \gamma_{N}$. Let $n \in\{1, \ldots, N\}$. As in the second paragraph of the proof of Lemma 5.1, we see that the $\rho$-length of $\gamma_{n}$ is at least $e^{n}-e^{n-1}=e^{n-1}(e-1)$. Every tile $t$ in $\mathcal{S}(X)$ which meets $\gamma_{n}$ is contained in $C_{0} \cup \cdots \cup C_{n}$. Hence the $\rho^{\prime}$-weight of every tile which meets $\gamma_{n}$ is at least $e^{1-n} /(e-1)$ times the $\rho$-weight of that tile. Hence the $\rho^{\prime}$-length of $\gamma_{n}$ is at least

$$
e^{n-1}(e-1) \frac{e^{1-n}}{e-1}=1
$$

Thus the $\rho^{\prime}$-length of $\gamma$ is at least $N /(L+1)$, and so $H\left(R_{i}, \rho^{\prime}\right) \geq N /(L+1)$.

In this paragraph we estimate the $\rho^{\prime}$-area $A\left(R_{i}, \rho^{\prime}\right)$ of $R_{i}$. Since every tile in $\mathcal{S}(X)$ of positive $\rho^{\prime}$-weight meets $D_{N}$,

$$
A\left(R_{i}, \rho^{\prime}\right) \leq A\left(D_{N}, \rho^{\prime}\right) \leq \sum_{n=0}^{N} A\left(D_{n}, \rho\right)\left(\frac{e^{1-n}}{e-1}\right)^{2}
$$

Let $t \in C_{0} \cup \cdots \cup C_{N}$. Since the tiles in $\mathcal{S}(X)$ are almost round $(M)$, there exist concentric circular disks $C$ and $D$ with Euclidean diameters $c$ and $d$, respectively, such that $C \subseteq t \subseteq D$ and $d \leq M c$. Hence

$$
\rho^{2}(t) \leq d^{2} \leq M^{2} c^{2}=M^{2} \frac{4}{\pi} A(C)
$$

where $A(C)$ is the Lebesgue area of $C$. Let $n \in\{0, \ldots, N\}$. The choice of $L$ implies that every tile in $\mathcal{S}(X)$ which meets $D_{n}$ is contained in $D_{n+L}$. Hence the $\rho$-area of $D_{n}$ is at most $4 M^{2} / \pi$ times the Lebesgue area of $D_{n+L}$ :

$$
A\left(D_{n}, \rho\right) \leq 4 M^{2} e^{2(n+L)} .
$$

Hence we may choose the constant $M$ so large that

$$
A\left(D_{n}, \rho\right)\left(\frac{e^{1-n}}{e-1}\right)^{2} \leq 4 M^{2} e^{2(n+L)}\left(\frac{e^{1-n}}{e-1}\right)^{2}=4 M^{2}\left(\frac{e^{L+1}}{e-1}\right)^{2} \leq M^{3} .
$$

Thus

$$
A\left(R_{i}, \rho^{\prime}\right) \leq \sum_{n=0}^{N} A\left(D_{n}, \rho\right)\left(\frac{e^{1-n}}{e-1}\right)^{2} \leq \sum_{n=0}^{N} M^{3}=(N+1) M^{3}
$$

Thus

$$
M\left(R_{i}, \mathcal{S}(X)\right) \geq \frac{H\left(R_{i}, \rho^{\prime}\right)^{2}}{A\left(R_{i}, \rho^{\prime}\right)} \geq \frac{N^{2}}{(L+1)^{2}} \frac{1}{(N+1) M^{3}} .
$$

Since $N$ can be taken arbitrarily large, it follows that $\lim _{i \rightarrow \infty} M\left(R_{i}, \mathcal{S}(X)\right)=\infty$.

This proves Theorem 5.5 . 
Theorem 5.6. Let $\mathcal{R}$ be a bounded valence finite subdivision rule whose mesh approaches 0 . Let $X$ be a bounded valence hyperbolic $\mathcal{R}$-complex. Let $D$ be an open topological disk in $X$. Then there exists a positive real number $K(D)$ such that if $R$ is a ring in $X$ surrounding $D$, then $M(R, \mathcal{S}(X)) \leq K(D)$. Furthermore, if $D_{1} \subseteq D_{2} \subseteq D_{3} \subseteq \ldots$ are open topological disks in $X$ such that $\bigcup_{i=1}^{\infty} D_{i}=X$, then $\lim _{i \rightarrow \infty} K\left(D_{i}\right)=0$.

Proof. To prove Theorem 5.6 we may replace $X$ by its uniformization. So we assume that $X$ is the open unit disk. Theorem 4.4 implies that the tiles of $X$ are almost round $(M)$ for some positive real number $M$. Let $d$ be the Euclidean diameter of $D$. Let $a$ be the Lebesgue area of the union of all tiles in $\mathcal{S}(X)$ which are not contained in $D$.

Let $R$ be a ring in $X$ surrounding $D$. Lemma 5.1 implies that

$$
m(R, \mathcal{S}(X)) \leq \frac{4 M^{2} A}{\pi \Delta^{2}},
$$

where $A$ is the Lebesgue area of the union of the tiles $t$ of $X$ for which the Lebesgue area of $t \cap R$ is not 0 and $\Delta$ is the Euclidean diameter of the inner boundary component of $R$. Since the tiles $t$ of $X$ which meet $R$ are not contained in $D$, it follows that $A \leq a$. Clearly $\Delta \geq d$. Thus

$$
m(R, \mathcal{S}(X)) \leq \frac{4 M^{2} a}{\pi d^{2}}
$$

Now the bounded valence theorem [4, Theorem 1.6] implies that there exists a positive integer $L$, independent of $D$, such that $M(R, \mathcal{S}(X)) \leq L m(R, \mathcal{S}(X))$. So $M(R, \mathcal{S}(X)) \leq 4 L M^{2} a / \pi d^{2}$. This proves the first statement of Theorem [5.6] The second statement of Theorem 5.6 follows from the fact that in the limit, the area $a$ goes to 0 while the Euclidean diameter $d$ is bounded from 0 .

This proves Theorem 5.6.

Corollary 5.7. Let $\mathcal{R}$ be a bounded valence finite subdivision rule whose mesh approaches 0 . Let $X$ be a bounded valence $\mathcal{R}$-complex which is homeomorphic to $\mathbb{R}^{2}$. Suppose that $S_{\mathcal{R}}$ has a partial conformal structure $\mathcal{A}$ such that the pullback of $\mathcal{A}$ to $X$ determines a conformal structure on $X$ and the pullback of $\mathcal{A}$ to $\mathcal{R}(X)$ determines a conformal structure on $\mathcal{R}(X)$. Then $X$ is parabolic, respectively hyperbolic, if and only if $\mathcal{R}(X)$ is parabolic, respectively hyperbolic.

Proof. This follows easily from Theorem 5.5. Theorem [5.6, and the bounded overlap theorem [8, Theorem 4.3.1].

Remark 5.8. Let $\mathcal{R}$ be a bounded valence finite subdivision rule whose mesh approaches 0 . Let $X$ be a bounded valence $\mathcal{R}$-complex which is homeomorphic to $\mathbb{R}^{2}$. In Section 3 we define $X$ to be either parabolic or hyperbolic by means of a partial conformal structure on $S_{\mathcal{R}}$. Theorems 5.5 and 5.6 show that this definition is independent of the partial conformal structure used.

\section{EXPANSION MAP APPROXIMATIONS}

Let $\mathcal{R}$ be a finite subdivision rule, and let $X$ be an expansion $\mathcal{R}$-complex with expansion map $\varphi$. Let $d$ be the skinny path pseudometric on $X$, as defined in the paragraph before Lemma 2.7. In the paragraph before Lemma 2.7 we defined a pseudometric $d_{n}$ on $X$ for every nonnegative integer $n$ so that if $x, y \in X$, then 
$d_{n}(x, y)=d\left(\varphi^{n}(x), \varphi^{n}(y)\right)$. We now allow $n$ to be any integer. Thus if $n \in \mathbb{Z}$ and if $x, y \in X$, then $d_{n}(x, y)$ is the smallest nonnegative integer $m$ such that there exist tiles $t_{0}, \ldots, t_{m}$ of $X$ for which $\varphi^{n}(x) \in t_{0}, \varphi^{n}(y) \in t_{m}$, and $t_{i} \cap t_{i+1} \neq \emptyset$ for $i \in\{0, \ldots, m-1\}$. If $n$ is a nonnegative integer, then $d_{n}$ is the skinny path pseudometric for the complex $\mathcal{R}^{n}(X)$. Two immediate facts are that $d_{n}(x, y)=$ $d_{n-1}(\varphi(x), \varphi(y))$ and $d_{n-1}(x, y) \leq d_{n}(x, y)$ for every $x, y \in X$ and every integer $n$.

Our next result states that if a function $\psi$ uniformly approximates an expansion map $\varphi$ with respect to the skinny path pseudometric, then the sequence of functions $\left\{\varphi^{-n} \circ \psi^{n}\right\}$ converges uniformly on compact subsets.

Theorem 6.1. Let $\mathcal{R}$ be a bounded valence finite subdivision rule whose mesh approaches 0 . Let $X$ be an expansion $\mathcal{R}$-complex with expansion map $\varphi$ and skinny path pseudometric d. Suppose that $\psi: X \rightarrow X$ is a function such that there exists a positive integer $K$ with the property that $d(\varphi(x), \psi(x)) \leq K$ for every $x \in X$. Then

(1) there exists a positive integer $L$ for which $d_{-n}\left(\varphi^{n}(x), \psi^{n}(x)\right) \leq L$ for every $x \in X$ and every nonnegative integer $n$, and

(2) the sequence of functions $\left\{\varphi^{-n} \circ \psi^{n}\right\}$ converges to a function from $X$ to $X$, the convergence being uniform on compact subsets.

Example 6.2. Before proving Theorem 6.1, we illustrate it with an example. We define a finite subdivision rule $\mathcal{R}$ so that $S_{\mathcal{R}}$ is a torus with one vertex, two edges, and one tile, which is a quadrilateral. Opposite sides of the quadrilateral are identified in the usual way to obtain a torus. Figure 9 determines $\mathcal{R}$ up to isomorphism by showing how the tile type $t$ of the quadrilateral subdivides. We orient $t$ and mark a vertex of $t$ so that every tile of $\mathcal{R}(t)$ is identified with $t$ in a way which preserves orientation and marked vertices. One checks that $\mathcal{R}$ has bounded valence. One also checks that the mesh of $\mathcal{R}$ approaches 0 combinatorially, and so [5. Theorem 2.3] implies that $\mathcal{R}$ can be defined so that its mesh approaches 0 .

There exists an expansion $\mathcal{R}$-complex $X$ which is combinatorially equivalent to the usual tiling of the plane by squares. The expansion map $\varphi$ maps every square to the union of five squares, four of which have an edge in common with the fifth. Figure 10 shows part of $X$. The thick edges indicate the image of the 1-skeleton of $X$ under $\varphi$. We identify $X$ with $\mathbb{C}$ so that the fixed point of $\varphi$ is 0 and the vertices of $X$ have the form $a+b i$, where $a$ and $b$ are odd integers. We also assume that $\varphi(1+i)=1+3 i$. Let $\psi: \mathbb{C} \rightarrow \mathbb{C}$ be the function which multiplies every element of $\mathbb{C}$ by $2+i$. The dashed edges in Figure 10 indicate the images of the 1-skeleton of $X$ under $\psi$. One checks that $\varphi(v)=\psi(v)$ for every vertex $v$ of $X$. This implies that there exists a positive integer $K$ such that $d(\varphi(x), \psi(x)) \leq K$ for every $x \in X$. Thus the assumptions of Theorem 6.1 are satisfied.

Statement 2 of Theorem 6.1 states that the sequence of functions $\left\{\varphi^{-n} \circ \psi^{n}\right\}$ converges to a function from $X$ to $X$. Figure11 illustrates the action on $X$ of the first few functions in the sequence $\left\{\varphi^{-n} \circ \psi^{n}\right\}$ as well as the action of the limit function. The first polygon, $S$, is the tile of $X$ which contains 0 . The function $\varphi^{-1} \circ \psi$ maps the second polygon to $S$. In the same way. the functions $\varphi^{-2} \circ \psi^{2}$, $\varphi^{-3} \circ \psi^{3}$, and $\varphi^{-4} \circ \psi^{4}$ map the third, fourth, and fifth polygons to $S$. The limit function maps the last region to $S$.

Proof. According to Lemma 2.7. because the mesh of $\mathcal{R}$ approaches 0 there exists a positive integer $N$ with the following property. Let $Y$ be an $\mathcal{R}$-complex and let $x$ 

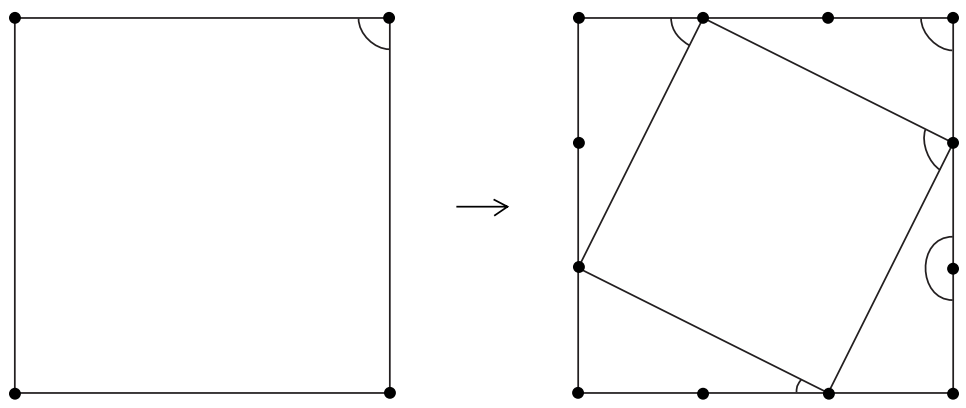

Figure 9 . The subdivision of the tile type $t$

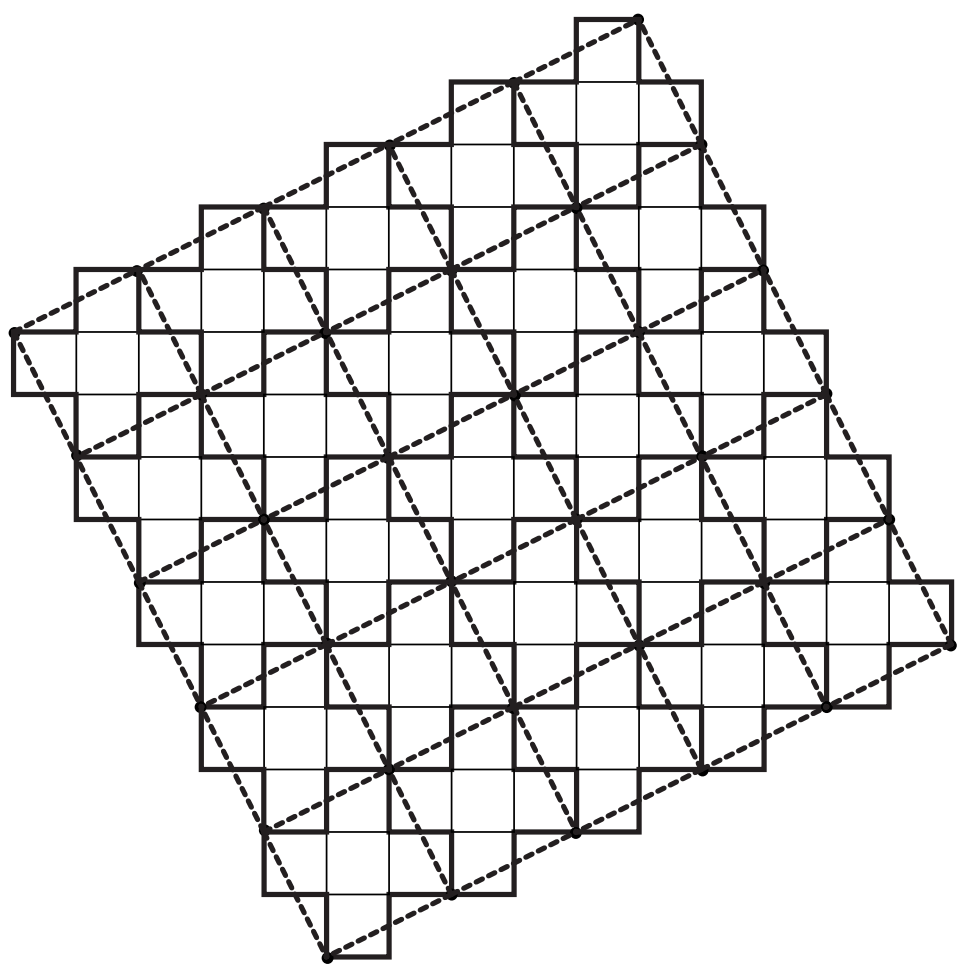

Figure 10. The expansion complex $X$

and $y$ be points in $Y$ such that the skinny path distance between $x$ and $y$ is at least 2. Then the skinny path distance between $x$ and $y$ relative to $\mathcal{R}^{N}(Y)$ is at least 2 times the skinny path distance between $x$ and $y$ relative to $Y$. We see by induction for every positive integer $k$ that the skinny path distance between $x$ and $y$ relative to $\mathcal{R}^{k N}(Y)$ is at least $2^{k+1}$. It follows that there exists a positive integer $p$ such that the skinny path distance between $x$ and $y$ relative to $\mathcal{R}^{p}(Y)$ is at least $p K+2$. Thus if $x, y \in X$ and $d_{n}(x, y) \leq p K+1$ for some integer $n$, then $d_{n-p}(x, y) \leq 1$.

In this paragraph we prove by induction that $d_{-n}\left(\varphi^{n}(x), \psi^{n}(x)\right) \leq n K$ for every $x \in X$ and for every positive integer $n$. First suppose that $n=1$. We have assumed 

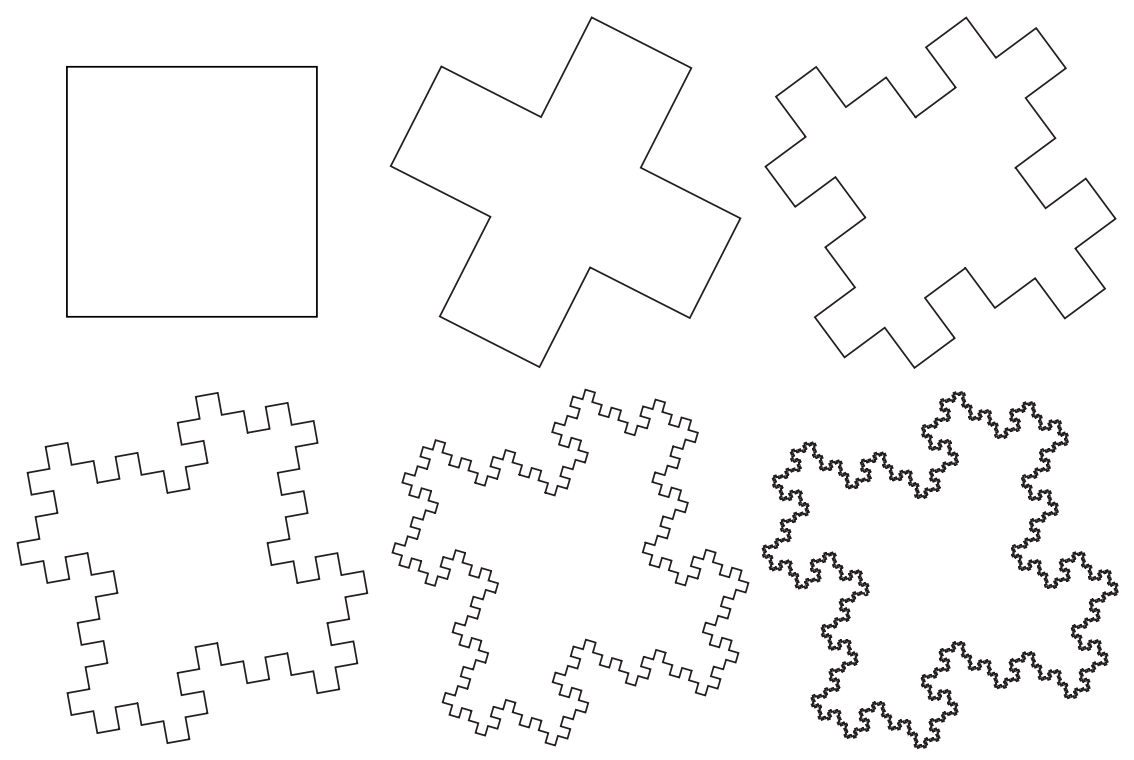

Figure 11. The action on $X$ of the functions $\varphi^{-n} \circ \psi^{n}$

that $d(\varphi(x), \psi(x)) \leq K$ for every $x \in X$. Since $d(w, z)=d_{0}(w, z) \geq d_{-1}(w, z)$ for every $w, z \in X$, it follows that $d_{-1}(\varphi(x), \psi(x)) \leq K$ for every $x \in X$. So let $n \geq 2$ be an integer, and suppose that

$$
d_{1-n}\left(\varphi^{n-1}(x), \psi^{n-1}(x)\right) \leq(n-1) K
$$

for every $x \in X$. Let $x \in X$. It follows that

$$
d_{-n}\left(\varphi^{n}(x), \varphi\left(\psi^{n-1}(x)\right)\right)=d_{1-n}\left(\varphi^{n-1}(x), \psi^{n-1}(x)\right) \leq(n-1) K .
$$

The previous case $(n=1)$ applied to the point $\psi^{n-1}(x)$ yields

$$
d_{-n}\left(\varphi\left(\psi^{n-1}(x)\right), \psi\left(\psi^{n-1}(x)\right)\right) \leq d_{-1}\left(\varphi\left(\psi^{n-1}(x)\right), \psi\left(\psi^{n-1}(x)\right)\right) \leq K .
$$

The triangle inequality now implies that $d_{-n}\left(\varphi^{n}(x), \psi^{n}(x)\right) \leq n K$, as desired.

We complete the proof of statement 1 in this paragraph. Set $L=p K+1$. Let $x \in X$, and let $n$ be a nonnegative integer. Statement 1 is trivially true if $n=0$, so suppose that $n>0$. Let $q$ and $r$ be the nonnegative integers for which $n=p q+r$ with $1 \leq r \leq p$. We prove by induction on $m \in\{0, \ldots, q\}$ that

$$
d_{-p m-r}\left(\varphi^{p m+r}\left(\psi^{p(q-m)}(x)\right), \psi^{n}(x)\right) \leq L .
$$


To begin, note that the previous paragraph implies that

$$
d_{-r}\left(\varphi^{r}\left(\psi^{p q}(x)\right), \psi^{r}\left(\psi^{p q}(x)\right) \leq r K .\right.
$$

Hence line (6.3) is true if $m=0$. Now suppose that line (6.3) is true for $m=k \in$ $\{0, \ldots, q-1\}$. Line (6.3) and the conclusion of the first paragraph of the proof of Theorem 6.1 imply that

$$
d_{-p(k+1)-r}\left(\varphi^{p k+r}\left(\psi^{p(q-k)}(x)\right), \psi^{n}(x)\right) \leq 1 .
$$

The previous paragraph implies that

$$
d_{-p}\left(\varphi^{p}\left(\psi^{p(q-k-1)}(x)\right), \psi^{p}\left(\psi^{p(q-k-1)}(x)\right)\right) \leq p K .
$$

Hence

$$
d_{-p(k+1)-r}\left(\varphi^{p(k+1)+r}\left(\psi^{p(q-k-1)}(x)\right), \varphi^{p k+r}\left(\psi^{p(q-k)}(x)\right)\right) \leq p K .
$$

Lines (6.4) and (6.5) and the triangle inequality imply that line (6.3) is true for $m=$ $k+1$. Thus line (6.3) is true for $m=q$, which proves statement 1 of Theorem 6.1.

We now turn to the proof of statement 2. For convenience, we assume that $X \subseteq \mathbb{R}^{2}$. Let $C$ be a compact subset of $X$. By statement 1 , there exists a positive integer $L$ for which $d_{-n}\left(\varphi^{n}(x), \psi^{n}(x)\right) \leq L$ for every nonnegative integer $n$ and every $x \in C$. Hence $d\left(x, \varphi^{-n}\left(\psi^{n}(x)\right)\right) \leq L$ for every nonnegative integer $n$ and every $x \in C$. Thus there exists a compact subset $C^{\prime}$ of $X$ which contains every sequence $\left\{\varphi^{-n}\left(\psi^{n}(x)\right)\right\}$ for $x \in C$. Let $\epsilon$ be a positive real number. Because the mesh of $\mathcal{R}$ approaches 0 , there exists a positive integer $m$ such that if $y, z \in C^{\prime}$ with $d_{m}(y, z) \leq L$, then $|y-z|<\epsilon$. Let $n$ be an integer with $n \geq m$. Statement 1 implies for every $x \in C$ that

$$
d_{m-n}\left(\varphi^{n-m}\left(\psi^{m}(x)\right), \psi^{n-m}\left(\psi^{m}(x)\right)\right) \leq L,
$$

hence

$$
d_{m}\left(\varphi^{-m}\left(\psi^{m}(x)\right), \varphi^{-n}\left(\psi^{n}(x)\right)\right) \leq L
$$

and so

$$
\left|\varphi^{-m}\left(\psi^{m}(x)\right)-\varphi^{-n}\left(\psi^{n}(x)\right)\right|<\epsilon .
$$

This implies that the sequences $\left\{\varphi^{-n}\left(\psi^{n}(x)\right)\right\}$ are uniformly Cauchy for $x \in C$. This proves statement 2 of Theorem 6.1 .

The proof of Theorem 6.1 is complete.

We next show that under the further assumption that the mesh of the sequence of tilings $\left\{\psi^{-n}(\mathcal{S}(X))\right\}$ locally approaches $0, \psi$ is the expansion map for an expansion complex $X^{\prime}$ that is topologically conjugate to $X$.

Theorem 6.6. Let $\mathcal{R}$ be a bounded valence finite subdivision rule whose mesh approaches 0 . Let $X$ be an expansion $\mathcal{R}$-complex with expansion map $\varphi$ and skinny path pseudometric d. Suppose that $\psi: X \rightarrow X$ is a homeomorphism such that there exists a positive integer $K$ for which $d(\varphi(x), \psi(x)) \leq K$ for every $x \in X$. Furthermore, suppose that the mesh of the sequence of tilings $\left\{\psi^{-n}(\mathcal{S}(X))\right\}$ locally approaches 0 . Then there exists an expansion $\mathcal{R}$-complex $X^{\prime}$ whose underlying space equals the underlying space of $X$ with expansion map $\psi$ such that $\mathcal{S}\left(X^{\prime}\right)$ has bounded overlap with $\mathcal{S}(X)$. Furthermore, the sequence of functions $\left\{\psi^{-n} \circ \varphi^{n}\right\}$ converges to an $\mathcal{R}$-isomorphism $\rho: X \rightarrow X^{\prime}$ with $\rho \circ \varphi=\psi \circ \rho$ and $d(x, \rho x)$ is globally bounded for $x \in X$. 
Proof. The assumptions of Theorem 6.1 are satisfied, so its conclusions hold. With the additional assumptions here, we will prove that the sequence of functions $\left\{\psi^{-n} \circ \varphi^{n}\right\}$ also converges to a function from $X$ to $X$. For convenience, we assume that $X \subseteq \mathbb{R}^{2}$.

Let $C$ be a compact subset of $X$. By statement 1 of Theorem 6.1, there exists a positive integer $L$ such that $d_{-n}\left(\varphi^{n}(x), \psi^{n}(x)\right) \leq L$ for every $x \in X$ and every nonnegative integer $n$. Hence $d\left(x, \varphi^{-n}\left(\psi^{n}(x)\right)\right) \leq L$ for every $x \in X$ and every nonnegative integer $n$. Given $y \in X$, choosing $x=\psi^{-n}\left(\varphi^{n}(y)\right)$ gives that $d\left(\psi^{-n}\left(\varphi^{n}(y)\right), y\right) \leq L$ for every nonnegative integer $n$. Thus there exists a compact subset $C^{\prime}$ of $X$ which contains every sequence $\left\{\psi^{-n}\left(\varphi^{n}(x)\right)\right\}$ for $x \in C$. Let $\epsilon$ be a positive real number. Because the mesh of $\left\{\psi^{-n}(\mathcal{S}(X))\right\}$ locally approaches 0 , there exists a positive integer $m$ such that if $u, v \in C^{\prime}$ and the skinny path distance relative to $\psi^{-m}(\mathcal{S}(X))$ between $u$ and $v$ is at most $L$, then $|u-v|<\epsilon$. Let $n$ be an integer with $n \geq m$, and let $x \in C$. Let $y=\varphi^{m}(x)$. Then, as before in this paragraph, $d\left(\psi^{m-n}\left(\varphi^{n-m}(y)\right), y\right) \leq L$, so $d\left(\psi^{m-n}\left(\varphi^{n}(x)\right), \varphi^{m}(x)\right) \leq L$. This implies that the skinny path distance relative to $\psi^{-m}(\mathcal{S}(X))$ between $u=\psi^{-n}\left(\varphi^{n}(x)\right)$ and $v=\psi^{-m}\left(\varphi^{m}(x)\right)$ is at most $L$, and so $\left|\psi^{-n}\left(\varphi^{n}(x)\right)-\psi^{-m}\left(\varphi^{m}(x)\right)\right|<\epsilon$. Hence the sequences $\left\{\psi^{-n}\left(\varphi^{n}(x)\right)\right\}$ are uniformly Cauchy for $x \in C$. This proves that the sequence of functions $\left\{\psi^{-n} \circ \varphi^{n}\right\}$ converges to a function $\rho: X \rightarrow X$. Since the sequence $\left\{\varphi^{-n} \circ \psi^{n}\right\}$ also converges, it is easy to see that $\rho$ is a homeomorphism and that $\rho \circ \varphi=\psi \circ \rho$. It is now a straightforward matter to define an $\mathcal{R}$-complex $X^{\prime}$ whose underlying space equals the underlying space of $X$ such that $\rho: X \rightarrow X^{\prime}$ is an $\mathcal{R}$-isomorphism and $\psi$ is the expansion map of $X^{\prime}$.

It remains to prove that $\mathcal{S}(X)$ and $\mathcal{S}\left(X^{\prime}\right)$ have bounded overlap and that $d(x, \rho x)$ is globally bounded for $x \in X$. We have seen for every $y \in X$ and every nonnegative integer $n$ that $d\left(\psi^{-n}\left(\varphi^{n}(y)\right), y\right) \leq L$. Hence $d(\rho(y), y) \leq L$ for every $y \in X$. Since $X$ has bounded valence, there is a constant $M$ such that each tile of $X^{\prime}$ intersects at most $M$ tiles of $X$ and each tile of $X$ intersects at most $M$ tiles of $X^{\prime}$.

This completes the proof of Theorem 6.6 .

The assumption in Theorem 6.6 about the mesh of the sequence of tilings locally approaching 0 is inconvenient. The next result shows that it holds in all cases currently of interest to us.

Theorem 6.7. Let $\mathcal{R}$ be a bounded valence finite subdivision rule whose mesh approaches 0 . Let $X$ be an expansion $\mathcal{R}$-complex with expansion map $\varphi$ and skinny path pseudometric d. Suppose that some partial conformal structure on $S_{\mathcal{R}}$ determines a conformal structure on $X$. Suppose that $\psi: X \rightarrow X$ is a conformal homeomorphism such that there exists a positive integer $K$ for which $d(\varphi(x), \psi(x)) \leq K$ for every $x \in X$. Then $X$ is parabolic and the mesh of the sequence of tilings $\left\{\psi^{-n}(\mathcal{S}(X))\right\}$ locally approaches 0 .

Proof. We begin by investigating the dynamics of $\psi$. Lemma 2.9 implies that $\varphi$ fixes a point $p \in X$. Statement 1 of Theorem 6.1 implies that there exists a positive integer $L$ for which $d_{-n}\left(\varphi^{n}(x), \psi^{n}(x)\right) \leq L$ for every $x \in X$ and every nonnegative integer $n$. Hence $d\left(x, \varphi^{-n}\left(\psi^{n}(x)\right)\right) \leq L$ for every $x \in X$ and every nonnegative integer $n$. Since $\varphi$ expands, if the sequence $\left\{\psi^{n}(y)\right\}$ is bounded relative to $d$ for some $y \in X$, then $d\left(\varphi^{-n}\left(\psi^{n}(y)\right), p\right)=0$ for $n \gg 0$. The triangle inequality applied to this and the previous inequality with $x=y$ shows that $d(y, p) \leq L$. Thus there exists $y \in X$ such that $\left\{\psi^{n}(y)\right\}$ is unbounded relative to $d$. On the other hand, taking 
$x=\psi^{-n}(p)$, we see that $d\left(\psi^{-n}(p), \varphi^{-n}(p)\right) \leq L$ for every nonnegative integer $n$. Hence $d\left(\psi^{-n}(p), p\right) \leq L$ for every nonnegative integer $n$. So the sequence $\left\{\psi^{-n}(p)\right\}$ is bounded relative to $d$. We have proved the following basic fact concerning the dynamics of $\psi$. The positive powers of $\psi$ applied to some element of $X$ do not lie in a bounded set and the negative powers of $\psi$ applied to another element of $X$ lie in a bounded set. No conformal automorphism of the open unit disk has this property. Thus $X$ is parabolic. Hence we may, and do, assume that the underlying space of $X$ is $\mathbb{C}$ and that $\psi(z)=a z+b$ for some $a, b \in \mathbb{C}$. Now one checks that the preceding basic fact concerning the dynamics of $\psi$ implies that $|a|>1$. Because $a \neq 1$, the function $\psi$ fixes some complex number. We may choose coordinates so that $\psi(0)=0$. Thus $\psi(z)=a z$ for some $a \in \mathbb{C}$ with $|a|>1$.

In this paragraph we show that it suffices to prove Theorem 6.7 for $\mathcal{R}^{N}$, where $N$ is any positive integer. So let $N$ be a positive integer. Then $\mathcal{R}^{N}$ is a bounded valence finite subdivision rule whose mesh approaches 0 . We have that $X$ is an expansion $\mathcal{R}^{N}$-complex with expansion map $\varphi^{N}$ and skinny path pseudometric $d$. Moreover, from the previous paragraph we have that $\psi^{N}: X \rightarrow X$ is a conformal homeomorphism such that there exists a positive integer $L$ for which $d_{-N}\left(\varphi^{N}(x), \psi^{N}(x)\right) \leq L$ for every $x \in X$. It follows that there exists a positive integer $M$ such that $d\left(\varphi^{N}(x), \psi^{N}(x)\right) \leq M$ for every $x \in X$. Hence the hypotheses of Theorem 6.7 for $\mathcal{R}, X, \varphi$ and $\psi$ imply the corresponding properties for $\mathcal{R}^{N}, X, \varphi^{N}$ and $\psi^{N}$. The conclusion of Theorem 6.7 for the latter case implies that if $B$ is a closed metric ball in $\mathbb{C}$ centered at 0 , then the mesh of the sequence of tilings $\left\{\psi^{-n N}(\mathcal{S}(X))\right\}$ approaches 0 on $B$. Because $\psi(z)=a z$ for every $z \in \mathbb{C}$, it follows that the mesh of the sequence of tilings $\left\{\psi^{-n}(\mathcal{S}(X))\right\}$ approaches 0 on $\psi^{-N}(B)$.

Thus to prove Theorem 6.7, we may replace $\mathcal{R}$ by $\mathcal{R}^{N}$ for any positive integer $N$. We choose $N$ as in Lemma 2.7. Therefore in addition to the assumptions in Theorem 6.7, we also have that if $x$ and $y$ are points of $X$ such that $d(x, y) \geq 2$, then $d(\varphi(x), \varphi(y)) \geq 2 d(x, y)$.

Let $s$ be a tile of $X$, and let $t$ be a tile of $\varphi(s)$. In this paragraph we prove that $\operatorname{star}^{K+1}(t, X) \subseteq \psi\left(\operatorname{star}^{K}(s, X)\right)$. For this, let $x \in \operatorname{int}(s)$ such that $\varphi(x) \in \operatorname{int}(t)$ and let $y$ be a point in $X \backslash \operatorname{star}^{K}(s, X)$. Then $d(x, y) \geq K+1 \geq 2$. The new assumption from the last paragraph implies that $d(\varphi(x), \varphi(y)) \geq 2 K+2$. The last original assumption implies that $d(\varphi(y), \psi(y)) \leq K$. Combining the last two inequalities gives $d(\varphi(x), \psi(y)) \geq K+2$. This implies that $\operatorname{star}^{K+1}(t, X) \subseteq \psi\left(\operatorname{star}^{K}(s, X)\right)$, as desired.

In this paragraph we choose a subcomplex of $X$ and a real number. Let $S$ be a finite $\mathcal{R}$-subcomplex of $X$ which contains a seed of $X$. As in the first paragraph of this proof, there exists a positive integer $L$ for which $d\left(x, \varphi^{-n}\left(\psi^{n}(x)\right)\right) \leq L$ for every $x \in S$ and every nonnegative integer $n$. It follows that $\varphi^{-n}\left(\psi^{n}(S)\right) \subseteq \operatorname{star}^{L}(S, X)$ for every nonnegative integer $n$. Let $T=\operatorname{star}^{L}(S, X)$. Then $T$ is a finite subcomplex of $X$ containing $S$ such that $S \subseteq \psi^{-n}\left(\varphi^{n}(T)\right)$ for every nonnegative integer $n$. As in Theorem 4.6, for every tile $t$ of $X$ we let $\delta_{K}(t)$ be the Euclidean diameter of the circumscribing disk of $\operatorname{star}^{K}(t, X)$. Theorem 4.6 implies that there exists a real number $c$ with $0<c<1$ such that $\delta_{K}(t) \leq c \delta_{K+1}(t)$ for every tile $t$ of $X$. We choose $c$ so that $c \geq|a|^{-1}$, which is possible because $|a|>1$. Let $D$ be a real number such that $\delta_{K}(t) \leq D$ for every tile $t$ of $T$.

Since the complex $T$ contains a seed of $X$, we have that $X=\bigcup_{n=0}^{\infty} \varphi^{n}(T)$. In this paragraph we prove by induction on $n$ that, if $t$ is any tile of $X$ and $n$ is 
the least nonnegative integer such that $t \subseteq \varphi^{n}(T)$, then $\delta_{K}(t) \leq|a|^{n} c^{n} D$. This inequality holds for $n=0$ by the definition of $D$. So suppose that $n>0$ and that the inequality holds for $n-1$. Let $s$ be the tile of $X$ such that $t \subseteq \varphi(s)$. Then $s \subseteq \varphi^{n-1}(T)$. By induction we have that $\delta_{K}(s) \leq|a|^{n-1} c^{n-1} D$. Thus the Euclidean diameter of the circumscribing disk of $\psi\left(\operatorname{star}^{K}(s, X)\right)$ is at most $|a|^{n} c^{n-1} D$. We proved that $\operatorname{star}^{K+1}(t, X) \subseteq \psi\left(\operatorname{star}^{K}(s, X)\right)$, and so $\delta_{K+1}(t) \leq|a|^{n} c^{n-1} D$. Since $\delta_{K}(t) \leq c \delta_{K+1}(t)$, we have that $\delta_{K}(t) \leq|a|^{n} c^{n} D$, as desired.

Now let $n$ be a nonnegative integer, and let $t$ be a tile of $\varphi^{n}(T)$. According to the last paragraph, there exists an integer $m$ with $0 \leq m \leq n$ such that $\delta_{K}(t) \leq|a|^{m} c^{m} D$. But $|a|^{m} c^{m} D \leq|a|^{n} c^{n} D$ because $|a| c \geq 1$. So the circumscribing disk of $\psi^{-n}(t)$ has Euclidean diameter at most $c^{n} D$. Since $S \subseteq \psi^{-n}\left(\varphi^{n}(T)\right)$ for every nonnegative integer $n$, this implies that the mesh of the sequence of tilings $\left\{\psi^{-n}(\mathcal{S}(X))\right\}$ approaches 0 on $S$. Since $S$ is any finite $\mathcal{R}$-subcomplex of $X$ which contains a seed of $X$, it follows that the mesh of the sequence of tilings $\left\{\psi^{-n}(\mathcal{S}(X))\right\}$ locally approaches 0 .

This proves Theorem 6.7

Corollary 6.8. Let $\mathcal{R}$ be a bounded valence finite subdivision rule whose mesh approaches 0 . Let $X$ be an expansion $\mathcal{R}$-complex with expansion map $\varphi$ and skinny path pseudometric d. Suppose that some partial conformal structure on $S_{\mathcal{R}}$ determines a conformal structure on $X$ which allows us to identify $X$ with $\mathbb{C}$. Suppose that $\alpha$ is a complex number and $K$ is a positive integer such that $d(\varphi(z), \alpha z) \leq K$ for every $z \in \mathbb{C}$. Then $\varphi$ and the map $z \mapsto \alpha z$ are conjugate by means of a homeomorphism $\rho$ of $\mathbb{C}$ such that $d(z, \rho(z))$ is globally bounded for $z \in \mathbb{C}$.

Proof. This corollary follows from Theorems 6.6 and 6.7.

Remark 6.9. For a given finite subdivision rule $\mathcal{R}$ with mesh approaching 0 and expansion complex $X$, there can be at most one $\alpha \in \mathbb{C}$ satisfying the hypothesis of Corollary 6.8 To see this, suppose that $\alpha$ is a complex number as is Corollary 6.8 and that $\beta$ is also such a complex number. Theorem 6.6 implies that the function $\varphi(z)=\alpha z$ is the expansion map for an expansion $\mathcal{R}$-complex. Furthermore, the function $\psi(z)=\beta z$ lies within bounded combinatorial distance from $\varphi$. Thus Theorems 6.6 and 6.7 imply that the sequence of functions $\left\{\psi^{-n} \circ \varphi^{n}\right\}$ converges to a homeomorphism. In other words, the sequence of complex numbers $\left\{\left(\beta^{-1} \alpha\right)^{n}\right\}$ converges to a nonzero complex number. Thus $\beta^{-1} \alpha=1$, and hence $\alpha=\beta$. So if $\alpha, \beta \in \mathbb{C}$ with $|\alpha|,|\beta|>1$ and $\alpha \neq \beta$, then the maps $z \mapsto \alpha z$ and $z \mapsto \beta z$ are topologically conjugate but they cannot both be topologically conjugate to $\varphi$ by conjugacies that move points a bounded combinatorial distance.

Theorem 6.10. Let $\mathcal{R}$ be a bounded valence finite subdivision rule whose mesh approaches 0 . Let $X$ be an expansion $\mathcal{R}$-complex with expansion map $\varphi$ such that $X=\mathbb{R}^{2}$. Suppose that there exists an almost round tiling $\mathcal{T}$ of $\mathbb{R}^{2}$ which has bounded overlap with $\mathcal{S}(X)$. Furthermore suppose that $\varphi$ is an $\mathbb{R}$-linear map. Then $(X, \mathcal{R})$ is conformal if and only if either $\varphi$ is a scalar map or the eigenvalues of $\varphi$ are not real numbers.

Proof. Let $\rho: \mathbb{R}^{2} \rightarrow \mathbb{R}^{2}$ be an $\mathbb{R}$-linear isomorphism. We may use $\rho$ to define a new expansion $\mathcal{R}$-complex $X^{\prime}$ such that $\rho: X \rightarrow X^{\prime}$ is an $\mathcal{R}$-isomorphism and the expansion map of $X^{\prime}$ is $\rho \circ \varphi \circ \rho^{-1}$. A straightforward verification shows that $\rho$ preserves the property of almost roundness. Since $\mathcal{T}$ is an almost round tiling of 
$\mathbb{R}^{2}$ which has bounded overlap with $\mathcal{S}(X)$, it follows that $\rho(\mathcal{T})$ is an almost round tiling of $\mathbb{R}^{2}$ which has bounded overlap with $\mathcal{S}\left(X^{\prime}\right)$. Hence we may replace $\varphi$ by $\rho \circ \varphi \circ \rho^{-1}$. Hence we may replace the matrix of $\varphi$ with respect to the standard basis of $\mathbb{R}^{2}$ by any similar matrix.

First suppose that $\varphi$ has an eigenvalue $\lambda=a+i b$ with $a, b \in \mathbb{R}$ and $b \neq 0$. There exists a nonzero $\lambda$-eigenvector for $\varphi$ in $\mathbb{C}^{2}$. Let $u+i v$ be a nonzero $\lambda$-eigenvector for $\varphi$ with $u, v \in \mathbb{R}^{2}$. A standard argument shows that $\{u, v\}$ is a basis for $\mathbb{R}^{2}$ and that with respect to this basis the matrix of $\varphi$ is $\left(\begin{array}{cc}a & b \\ -b & a\end{array}\right)$. But when $\mathbb{R}^{2}$ is identified with $\mathbb{C}$ as usual, this is the matrix of the map $\psi: \mathbb{C} \rightarrow \mathbb{C}$ given by $\psi(z)=\bar{\lambda} z$ for every $z \in \mathbb{C}$. The previous paragraph shows that we may replace $\varphi$ by $\psi$.

Since the mesh of $\mathcal{R}$ approaches 0 , the mesh of the sequence $\left\{\varphi^{-n}(\mathcal{S}(X))\right\}$ of tilings locally approaches 0 . Since $\mathcal{T}$ has bounded overlap with $\mathcal{S}(X)$, it follows that the mesh of $\left\{\varphi^{-n}(\mathcal{T})\right\}$ locally approaches 0 . Since $\mathcal{T}$ is almost round and $\varphi$ is now given by multiplication by a nonzero complex number, it follows that $\varphi^{-n}(\mathcal{T})$ is almost round $(M)$ with respect to a fixed $M$ for every nonnegative integer $n$. Hence [3, Theorem 7.1] implies that the sequence $\left\{\varphi^{-n}(\mathcal{T})\right\}$ is conformal on every compact subset of $\mathbb{R}^{2}$. Now the bounded overlap theorem [8, Theorem 4.3.1] implies that $(X, \mathcal{R})$ is conformal.

Next suppose that the eigenvalues of $\varphi$ are real numbers. Then we may assume that the matrix of $\varphi$ with respect to the standard basis of $\mathbb{R}^{2}$ is in Jordan canonical form. The possibilities are as follows.

$$
\left(\begin{array}{cc}
\lambda & 0 \\
0 & \lambda
\end{array}\right) \quad\left(\begin{array}{cc}
\lambda & 0 \\
0 & \mu
\end{array}\right) \quad\left(\begin{array}{cc}
\lambda & 0 \\
1 & \lambda
\end{array}\right)
$$

We may assume that $|\lambda|<|\mu|$. Clearly $\lambda$ and $\mu$ are not 0 .

In the case of a scalar matrix, it is possible to prove that $(X, \mathcal{R})$ is conformal using the last paragraph of the argument used for the case in which the eigenvalues of $\varphi$ are not real.

Now suppose that the matrix of $\varphi$ with respect to the standard basis of $\mathbb{R}^{2}$ has the form $\left(\begin{array}{cc}\lambda & 0 \\ 0 & \mu\end{array}\right)$ with $|\lambda|<|\mu|$. Let $S$ be a ring in $\mathbb{R}^{2}$ surrounding 0 , and let $R$ be a ring in the interior of $S$ which separates the ends of $S$. Theorem 5.2 states that

$$
m\left(R, \mathcal{S}\left(\mathcal{R}^{n}(X)\right)\right) \leq K \frac{A\left(\varphi^{n}(S)\right)}{D\left(\varphi^{n}(S)\right)^{2}}
$$

for some positive real number $K$ and every nonnegative integer $n$. Because areas are multiplied by absolute values of determinants, we have that $A\left(\varphi^{n}(S)\right) \leq K_{1}|\lambda \mu|^{n}$ for some positive real number $K_{1}$ and for every nonnegative integer $n$. Because the inner boundary component of $S$ meets the $\mu$-eigenspace of $\varphi$, we have that $D\left(\varphi^{n}(S)\right) \geq K_{2}|\mu|^{n}$ for some positive real number $K_{2}$ and for every nonnegative integer $n$. Hence the moduli $m\left(R, \mathcal{S}\left(\mathcal{R}^{n}(X)\right)\right)$ degenerate to 0 . It follows that $(X, \mathcal{R})$ is not conformal.

Finally suppose that the matrix of $\varphi$ with respect to the standard basis of $\mathbb{R}^{2}$ has the form $\left(\begin{array}{cc}\lambda & 0 \\ 1 & \lambda\end{array}\right)$. For every nonnegative integer $n$,

$$
\left(\begin{array}{cc}
\lambda & 0 \\
1 & \lambda
\end{array}\right)^{n}=\left(\begin{array}{cc}
\lambda^{n} & 0 \\
n \lambda^{n-1} & \lambda^{n}
\end{array}\right)
$$


We argue as in the previous paragraph. In this case we have an area estimate of the form $A\left(\varphi^{n}(S)\right) \leq K_{1} \lambda^{2 n}$, and we have an estimate on Euclidean diameters of the form $D\left(\varphi^{n}(S)\right) \geq K_{2} n|\lambda|^{n}$. As before, the moduli $m\left(R, \mathcal{S}\left(\mathcal{R}^{n}(X)\right)\right)$ degenerate to 0 , and $(X, \mathcal{R})$ is not conformal.

This proves Theorem 6.10 .

The one-tile single valence finite subdivision rules of [7] provide an interesting class of examples to which Theorem 6.10 applies.

\section{ACKNOWLEDGMENT}

We thank the referee for many helpful comments.

\section{REFERENCES}

[1] M. Bestvina and G. Mess, The boundary of negatively curved groups, J. Amer. Math. Soc. 4, (1991), 469-481. MR 1096169 (93j:20076)

[2] P. L. Bowers and K. Stephenson, A "regular" pentagonal tiling of the plane, Conform. Geom. Dyn. 1 (1997), 58-68 (electronic). MR1479069 (99d:52016)

[3] J. W. Cannon, The combinatorial Riemann mapping theorem, Acta Math. 173 (1994), 155-234. MR.1301392 (95k:30046)

[4] J. W. Cannon, W. J. Floyd, and W. R. Parry, Sufficiently rich families of planar rings, Ann. Acad. Sci. Fenn. Math. 24 (1999), 265-304. MR1724092(2000k:20057)

[5] J. W. Cannon, W. J. Floyd, and W. R. Parry, Finite subdivision rules, Conform. Geom. Dyn. 5 (2001), 153-196 (electronic). MR1875951 (2002j:52021)

[6] J. W. Cannon, W. J. Floyd, R. Kenyon, and W. R. Parry, Constructing rational maps from subdivision rules, Conform. Geom. Dyn. 7 (2003), 76-102 (electronic). MR.1992038 (2004f:37062)

[7] J. W. Cannon, W. J. Floyd, and W. R. Parry, Expansion complexes for finite subdivision rules $I I$, preprint, available from http://www.math.vt.edu/people/floyd.

[8] J. W. Cannon and E. L. Swenson, Recognizing constant curvature discrete groups in dimension 3, Trans. Amer. Math. Soc. 350 (1998), 809-849. MR.1458317 (98i:57023)

[9] C. Caratheodory, Theory of Functions of a Complex Variable, Vol. II, Chelsea, New York, 1960.

[10] A. Douady and J. H. Hubbard, A proof of Thurston's topological characterization of rational functions, Acta Math. 171, (1993), 263-297. MR1251582 (94j:58143)

[11] H. Farkas and I. Kra, Riemann Surfaces, Springer-Verlag, New York Heidelberg Berlin, 1980. MR0583745 (82c:30067)

[12] D. Gabai, G. R. Meyerhoff, and N. Thurston, Homotopy hyperbolic 3-manifolds are virtually hyperbolic, J. Amer. Math. Soc. 7, (1994), 193-198. MR1205445 (94b:57016)

[13] G. M. Goluzin, Geometric Theory of Functions of a Complex Variable, American Mathematical Society, Providence, 1969. MR0247039 (40:308)

[14] M. Gromov, Hyperbolic groups, Essays in Group Theory, (S. M. Gersten, ed.), SpringerVerlag, New York Berlin Heidelberg, 1987, 75-264. MR0919829 (89e:20070)

[15] L. Mosher, Geometry of cubulated 3-manifolds, Topology 34, (1995), 789-814. MR.1362788 (97i:57017)

[16] Z. Nehari, Conformal Mapping, McGraw-Hill, New York Toronto London, 1952. MR0045823 (13:640h)

[17] G. Perelman, Entropy formula for the Ricci flow and its geometric applications, preprint, available from http://www.arXiv.org/abs/math.DG/0211159.

[18] G. Perelman, Finite extinction time for the solutions to the Ricci flow on certain threemanifolds, preprint, available from http://www.arXiv.org/abs/math.DG/0307245.

[19] G. Perelman, Ricci flow with surgery on 3-manifolds, preprint, available from http: //www.arXiv.org/abs/math.DG/0303109. 
[20] K. Stephenson, CirclePack, software, available from http://www.math.utk.edu/ ${ }^{\sim}$ kens.

[21] W. P. Thurston, Lecture notes, CBMS Conference, University of Minnesota at Duluth, 1983.

Department of Mathematics, Brigham Young University, Provo, Utah 84602

E-mail address: cannon@math.byu.edu

Department of Mathematics, Virginia Tech, Blacksburg, Virginia 24061

E-mail address: floyd@math.vt.edu

URL: http://www.math.vt.edu/people/floyd

Department of Mathematics, Eastern Michigan University, Ypsilanti, Michigan 48197

E-mail address: walter.parry@emich.edu 